\title{
Palladium-Catalyzed Multistep Reactions Involving Ring \\ Closure of 2-Iodophenoxyallenes and Ring Opening of \\ Bicyclic Alkenes
}

Kanniyappan Parthasarathy, Masilamani Jeganmohan and Chien-Hong Cheng*

Department of Chemistry, National Tsing Hua University, Hsinchu, 30013 Taiwan

chcheng@mx.nthu.edu.tw

\section{Supporting Information}

\section{Table of Contents}

Page S2 Experimental Section

Page S2 - S8 IR, $\quad$ I H, ${ }^{13} \mathrm{C}$ NMR and HRMS Data

Page S8 - S8 References

Page S9 - S34 Copy of ${ }^{1} \mathrm{H}$ and ${ }^{13} \mathrm{C}$ NMR Spectra 
General. All reactions were conducted under nitrogen atmosphere on a dual-manifold Schlenk line unless otherwise mentioned and in oven-dried glass wares. All solvents were dried according to known methods and distilled prior to use. ${ }^{1}$ Starting materials substituted iodoallenes $\mathbf{1} \mathbf{a}-\mathbf{i}^{2}{ }^{2}$ and substituted bicyclic alkenes $\mathbf{2} \mathbf{b}-\mathbf{d}^{3}$ were synthesized according to the literature procedures. $\mathrm{Pd}\left(\mathrm{PPh}_{3}\right)_{4},{ }^{4} \mathrm{PdCl}_{2}\left(\mathrm{PPh}_{3}\right)_{2},{ }^{5}$ and $\operatorname{Pd}(\mathrm{dba})_{2}{ }^{6}$ were prepared by reported procedures. Other reagents were commercially available and used as purchased.

\section{General Procedure for Palladium-Catalyzed Ring Closure and Ring Opening}

\section{Reactions:}

A $25 \mathrm{~mL}$ round-bottom side-arm flask containing $\mathrm{PdCl}_{2}\left(\mathrm{PPh}_{3}\right)_{2}(0.0500 \mathrm{mmol}), \mathrm{Zn}$ powder (1.5 mmol ) was evacuated and purged with nitrogen gas three times. To the flask were then added iodoallenes (1) (1.00 mmol), bicyclic alkenes (2) (1.20 mmol) and THF (3.0 mL) via syringes. The reaction mixture was allowed to stir at $80{ }^{\circ} \mathrm{C}$ for $16 \mathrm{~h}$. At the end of the reaction, the reaction mixture was diluted with $\mathrm{CH}_{2} \mathrm{Cl}_{2}$, filtered through Celite and silica gel, and the filtrate was concentrated. The crude residue was purified through a silica gel column using hexane and ethyl acetate as eluent to give pure ring opening product 3 .

Spectral data and copy of ${ }^{1} \mathrm{H}$ and ${ }^{13} \mathrm{C}$ NMR spectra of all compounds are listed below.

\section{2-(Benzo[b]furan-3-ylmethyl)-1,2-dihydro-1-naphthalenol (3a):}

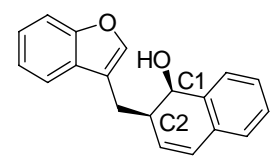

Colorless viscous oil; IR (neat): $3390 \mathrm{~cm}^{-1}(\mathrm{OH}) ;{ }^{1} \mathrm{H}$ NMR (500 MHz, $\mathrm{CDCl}_{3}$ ): $\delta 7.61$ - 7.45 (m, 3 H), 7.29 - 7.19 (m, 5 H), 7.13 (d, $J=7.5 . ~ H z, 1$ H), 6.58 (dd, $J=9.5,2.0$. Hz, $1 \mathrm{H}$ ), 5.86 (d, $J=10.0 \mathrm{~Hz}, 1 \mathrm{H})$ ), 4.54 (d, $J$ = 3.5 Hz, 1 H) (C1 proton), 3.23 (dd, 
$J=13.5,7.0 . \mathrm{Hz}, 1 \mathrm{H}), 2.99-2.90$ (m, $2 \mathrm{H})\left(\mathrm{C} 2\right.$ proton and a methylene proton); ${ }^{13} \mathrm{C}$ NMR (125 MHz, $\left.\mathrm{CDCl}_{3}\right): \delta 155.4,142.4,136.3,132.5,130.0,128.7,128.2,127.8$, 127.3, 126.7, 124.2, 122.3, 119.7, 118.0, 111.5, 69.8, 40.3, 23.3; HRMS (EI): calcd for $\mathrm{C}_{19} \mathrm{H}_{16} \mathrm{O}_{2}$ 276.1150, found 276.1147.

\section{2-(Benzo[b]furan-3-ylmethyl)-1,2-dihydro-1-triphenylenol (3b):}

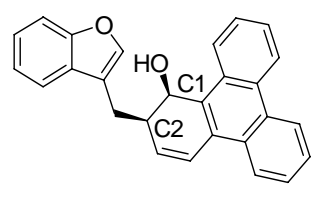

Pale yellow viscous oil; IR (neat): $3380 \mathrm{~cm}^{-1}(\mathrm{OH}) ;{ }^{1} \mathrm{H}$ NMR (500 MHz, $\left.\mathrm{CDCl}_{3}\right): \delta$ 8.70 (t, $J=8.0 \mathrm{~Hz}, 2 \mathrm{H}), 8.22(\mathrm{t}, J=4.5 \mathrm{~Hz}, 2 \mathrm{H}), 7.70-7.65(\mathrm{~m}, 6 \mathrm{H}), 7.62-7.59(\mathrm{~m}$, $1 \mathrm{H}), 7.52-7.41$ (m, $1 \mathrm{H}), 7.30$ (t, $J=7.0 \mathrm{~Hz}, 1 \mathrm{H}$ ), 7.22 (d, $J=7.0 \mathrm{~Hz}, 1 \mathrm{H}), 6.19$ (d, $J=9.5 \mathrm{~Hz}, 1 \mathrm{H}), 5.32$ (s, $1 \mathrm{H})$ (C1 proton), 3.42 - 3.39 (m, $1 \mathrm{H}), 3.23$ - 3.19 (m, $1 \mathrm{H})$, 3.08 - 3.01 (m, $1 \mathrm{H})$ (C2 proton); ${ }^{13} \mathrm{C}$ NMR (125 MHz, $\left.\mathrm{CDCl}_{3}\right): \delta$ 155.5, 142.5, 131.0, 130.6, 130.5, 129.8, 129.6, 128.6, 128.3, 127.3, 126.9, 126.8, 126.6, 126.4, 124.3, 124.1, 123.7, 123.4, 123.2, 123.0, 122.5, 119.7, 118.1, 111.6, 65.5, 40.4, 23.8; HRMS (EI): calcd for $\mathrm{C}_{27} \mathrm{H}_{20} \mathrm{O}_{2} 376.1463$, found 376.1465.

\section{6-(Benzo[b]furan-3-ylmethyl)-5,6-dihydronaphtho[2,3-d][1,3]dioxol-5-ol (3c):}

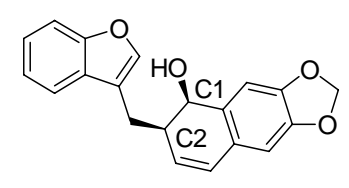

Yellowish viscous oil; IR (neat): $3395 \mathrm{~cm}^{-1}(\mathrm{OH}) ;{ }^{1} \mathrm{H}$ NMR (500 MHz, $\left.\mathrm{CDCl}_{3}\right)$ : $\delta$ 7.58 (d, $J=7.5 \mathrm{~Hz}, 1 \mathrm{H}), 7.53$ (s, $1 \mathrm{H}), 7.48$ (d, $J=8.5 \mathrm{~Hz}, 1 \mathrm{H}), 7.39$ - 7.30 (m, $2 \mathrm{H})$, 6.76 (s, 1 H), 6.62 (s, 1 H), 6.44 (dd, $J=13.0,9.5$ Hz, 1 H), 5.97 (s, 2 H), 5.75 - 5.72 (m, $1 \mathrm{H}), 4.40$ (d, $J=3.5 \mathrm{~Hz}, 1 \mathrm{H}$ ) (C1 proton), 3.17 (dd, $J=14.5,8.5 \mathrm{~Hz}, 1 \mathrm{H}), 2.95$ (dd, $J=14.5,6.0 \mathrm{~Hz}, 1 \mathrm{H}), 2.88-2.86$ (m, $1 \mathrm{H})$ (C2 proton); ${ }^{13} \mathrm{C}$ NMR (125 MHz, $\left.\mathrm{CDCl}_{3}\right): \delta 155.4,147.6,146.6,142.6,130.6,128.2,128.1,127.1,127.0,124.2,122.3$, 
119.7, 118.0, 111.5, 108.7, 107.2, 101.1, 69.9, 40.4, 23.4; HRMS (EI): calcd for $\mathrm{C}_{20} \mathrm{H}_{16} \mathrm{O}_{4} 320.1049$, found 320.1050.

Ethyl $N$-[2-(benzo[b]furan-3-ylmethyl)-1,2-dihydro-1-naphthalenyl]carbamate (3d):

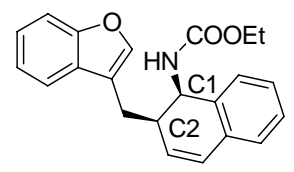

Colorless oil; IR (neat): $3409 \mathrm{~cm}^{-1}$ (NH) $\mathrm{cm}^{-1} ;{ }^{1} \mathrm{H}$ NMR (400 MHz, $\mathrm{CDCl}_{3}$ ): $\delta 7.54-$ 7.47 (m, 3 H), 7.35 (d, $J=6.8$ Hz, 1 H), 7.30 - 7.19 (m, 4 H), 7.09 (d, J= 7.2 Hz, 1 H), 6.54 (dd, $J=9.6,2.4 . \mathrm{Hz}, 1 \mathrm{H}$ ), 5.82 (d, $J=7.2,2.4 . \mathrm{Hz}, 1 \mathrm{H}$ ) ,5.16 (d, $J=10 \mathrm{~Hz}, 1$ H), 4.94 (dd, $J=10.0,4.8 \mathrm{~Hz}, 1 \mathrm{H})$ (C1 proton), 4.14 - 4.07 (m, 2 H), 3.09 - 3.03 (m, $2 \mathrm{H}), 2.85$ (q, $J=20.0,4.8 \mathrm{~Hz}, 1 \mathrm{H})(\mathrm{C} 2$ proton), 1.24 (q, $J=16.0,9.2 \mathrm{~Hz}, 3 \mathrm{H}) ;{ }^{13} \mathrm{C}$ NMR (100 MHz, $\left.\mathrm{CDCl}_{3}\right): \delta$ 156.2, 155.4, 142.3, 135.5, 132.4, 129.8, 128.2, 127.9, 127.4, 126.5, 124.2, 122.3, 119.5, 117.5, 111.5, 60.9, 51.5, 38.4, 23.7, 14.5; HRMS (EI): calcd for $\mathrm{C}_{22} \mathrm{H}_{21} \mathrm{NO}_{3}$ 347.1521, found 347.1523.

2-[(6-Methylbenzo[b]furan-3-yl)methyl]-1,2-dihydro-1-naphthalenol (3e):

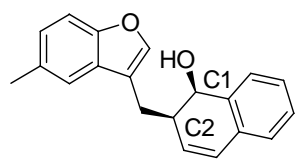

Pale yellow viscous oil; IR (neat): $3350 \mathrm{~cm}^{-1}(\mathrm{OH}) ;{ }^{1} \mathrm{H}$ NMR (400 MHz, $\left.\mathrm{CDCl}_{3}\right): \delta$ 7.50 (s, $1 \mathrm{H}$ ), 7.38 - 7.09 (m, 7 H), 6.57 (dd, $J$ = 9.6, 2.4.Hz, $1 \mathrm{H}$ ), 5.85 (dd, $J$ = 9.6, $1.2 \mathrm{~Hz}, 1 \mathrm{H}), 4.55$ (d, $J=3.6 \mathrm{~Hz}, 1 \mathrm{H})(\mathrm{C} 1$ proton), 3.18 (q, $J=17.2,6.0 \mathrm{~Hz}, 1 \mathrm{H})$ (C2 proton), 2.97 - 2.91 (m, $2 \mathrm{H}), 2.43$ (s, $3 \mathrm{H}), 1.66$ (d, $J=7.2 \mathrm{~Hz}, 1 \mathrm{H}) ;{ }^{13} \mathrm{C} \mathrm{NMR}$ (100 MHz, $\left.\mathrm{CDCl}_{3}\right): \delta$ 153.9, 142.5, 136.4, 132.6, 131.8, 130.1, 128.7, 128.3, 127.7, 127.3, 126.6, 125.4, 119.5, 117.7, 110.9, 69.9, 40.2, 23.3, 21.3; HRMS (EI): calcd for $\mathrm{C}_{20} \mathrm{H}_{18} \mathrm{O}_{2} 290.1307$, found 290.1305 .

2-(Naphtho[2,1-b]furan-1-ylmethyl)-1,2-dihydro-1-naphthalenol (3f): 


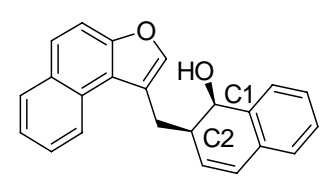

Colorless viscous oil; IR (neat): $3360 \mathrm{~cm}^{-1}(\mathrm{OH}) ;{ }^{1} \mathrm{H}$ NMR (400 MHz, $\mathrm{CDCl}_{3}$ ): $\delta 8.34$ (d, $J=8.0 \mathrm{~Hz}, 1 \mathrm{H}$ ), 7.95 (d, $J=7.6 \mathrm{~Hz}, 1 \mathrm{H}$ ), $7.74-7.66$ (m, $3 \mathrm{H}$ ), 7.57 (t, $J=7.6$ Hz, $1 \mathrm{H}), 7.47$ (t, $J=8.0 \mathrm{~Hz}, 1 \mathrm{H}), 7.29-7.20$ (m, $3 \mathrm{H}), 7.14$ (d, $J=7.2 \mathrm{~Hz}, 1 \mathrm{H}) 6.61$ (dd, $J=7.0,3.5 \mathrm{~Hz}, 1 \mathrm{H}$ ), 5.97 (dd, $J=10.0,3.0 \mathrm{~Hz}, 1 \mathrm{H}$ ), 4.60 (t, $J=4.8 \mathrm{~Hz}, 1 \mathrm{H}$ ) (C1 proton), 3.59 (dd, $J=14.8,8.0 \mathrm{~Hz}, 1 \mathrm{H}), 3.20$ (dd, $J=15.2$, 8.4. Hz,1 H), 3.12 3.08 (m, $1 \mathrm{H}$ ) (C2 proton), 1.80 (d, $J=7.2 \mathrm{~Hz}, 1 \mathrm{H}) ;{ }^{13} \mathrm{C}$ NMR (100 MHz, $\left.\mathrm{CDCl}_{3}\right): \delta$ 153.6, 142.1, 136.4, 132,5, 130.7, 129.9, 129.1, 128.6, 127.7, 127.6, 127.5, 126.6, 126.3, 125.5, 124.0, 123.1, 121.3, 119.8, 112.7, 69.9, 39.8, 25.2; HRMS: calcd for $\mathrm{C}_{23} \mathrm{H}_{18} \mathrm{O}_{2}$ 326.1307, found 326.1312.

\section{2-(1H-4-Isochromenylmethyl)-1,2,3,4-tetrahydro-1-naphthalenol (3g):}

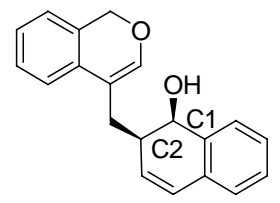

Pale yellow viscous oil; IR (neat): $3430 \mathrm{~cm}^{-1}(\mathrm{OH}) ;{ }^{1} \mathrm{H}$ NMR (400 MHz, $\left.\mathrm{CDCl}_{3}\right): \delta$ 7.33 - 7.13 (m, 7 H), 7.05 (d, $J=7.2$ Hz, 1 H), 6.63 (s, 1 H), 6.61 (d, $J=9.6$ Hz, 1 H), 5.91 (d, $J=9.2 \mathrm{~Hz}, 1 \mathrm{H}$ ), 5.03 (s, $2 \mathrm{H}$ ), 4.64 (s, $1 \mathrm{H}$ ) (C1 proton), 2.92 (dd, $J$ = 14.0, 7.6 Hz, 1H), 2.78 (m, $1 \mathrm{H}$ ) (C2 Proton), 2.62 (dd, $J=14.0,8.0 \mathrm{~Hz}, 1 \mathrm{H}) ;{ }^{13} \mathrm{C}$ NMR (100 MHz, $\left.\mathrm{CDCl}_{3}\right): \delta 144.2,136.4,132.7,131.0,130.2,128.9 .128 .6,128.1,127.7$, 127.1, 126.7, 126.6, 124.2, 120.3, 113.3, 70.0, 68.4, 38.8, 27.3; HRMS (EI): calcd for $\mathrm{C}_{20} \mathrm{H}_{18} \mathrm{O}_{2} 290.1307$, found 290.1308.

\section{2-[(5-Methoxy-1H-4-isochromenyl)methyl]-1,2,3,4-tetrahydro-1-naphthalenol} (3h): 


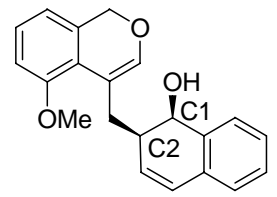

Pale yellow viscous oil; IR (neat): $3448 \mathrm{~cm}^{-1}(\mathrm{OH}) ;{ }^{1} \mathrm{H}$ NMR (400 MHz, $\left.\mathrm{CDCl}_{3}\right): \delta$ 7.25 - 7.08 (m, $6 \mathrm{H}), 6.78$ (d, $J=8.4 \mathrm{~Hz}, 1 \mathrm{H}), 6.67$ (d, $J=7.2 \mathrm{~Hz}, 1 \mathrm{H}), 6.57$ (s, $1 \mathrm{H}$ ), 6.52 (dd, $J=9.2,2.0 \mathrm{~Hz}, 1 \mathrm{H}), 4.89$ (d, $J=4.4 \mathrm{~Hz}, 2 \mathrm{H}), 4.57$ (d, $J=3.6 \mathrm{~Hz}, 1 \mathrm{H})(\mathrm{C} 1$ proton), 3.80 (s, $3 \mathrm{H}$ ), 3.10 (dd, $J=12.8,6.4,1 \mathrm{H}), 2.76$ - 2.74 (m, $2 \mathrm{H})$ (C2 proton and a methylene proton); ${ }^{13} \mathrm{C}$ NMR (125 $\left.\mathrm{MHz}, \mathrm{CDCl}_{3}\right): \delta 154.4,144.4,136.6$, 132.9, 131.9, 130.9, 128.5, 127.7, 127.6, 127.4, 126.7, 126.5, 119.6, 117.3, 114.8, 111.6, 70.3, 69.1, 55.4, 40.03, 30.73; HRMS (EI): calcd for $\mathrm{C}_{21} \mathrm{H}_{20} \mathrm{O}_{3} 320.1412$, found 320.1410.

\section{2-[(7-Methoxy-1H-4-isochromenyl)methyl]-1,2,3,4-tetrahydro-1-naphthalenol} (3i):

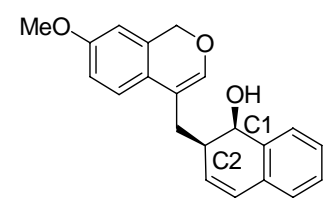

Brownish viscous oil; IR (neat): $3435 \mathrm{~cm}^{-1}(\mathrm{OH}) ;{ }^{1} \mathrm{H}$ NMR (500 MHz, $\left.\mathrm{CDCl}_{3}\right): \delta 7.27$ - 7.18 (m, 3 H), 7.10 - 7.06 (m, 3 H), 6.76 (dd, $J=8.0,2.0$ Hz,1 H), 6.60 (d, $J=3.0$ Hz, 1 H), 6.56 - 6.52 (m, 2 H), 5.88 (dd, $J=9.0,1.5 . \mathrm{Hz}, 1 \mathrm{H}), 4.96$ (d, $J=2.5 \mathrm{~Hz}, 2$ H), 4.60 (d, $J=4.0 \mathrm{~Hz}, 1 \mathrm{H}$ ) (C1 proton), 3.77 (s, $3 \mathrm{H}$ ), 2.86 (dd, $J=14.0,7.5 . \mathrm{Hz} 1$ H), 2.76 - 2.70 (m, $1 \mathrm{H}$ ) (C2 proton), 2.55 (dd, $J=17.5,7.5 \mathrm{~Hz}, 1 \mathrm{H}) ;{ }^{13} \mathrm{C}$ NMR (125 $\left.\mathrm{MHz}, \mathrm{CDCl}_{3}\right): \delta$ 158.6, 142.2, 136.5, 132.7, 130.8, 130.3, 128.6, 127.6, 127.1, 126.6, 123.9, 121.6, 113.2, 112.7, 110.5, 70.1, 68.4, 55.3, 38.9, 27.4; HRMS (EI): calcd for $\mathrm{C}_{21} \mathrm{H}_{20} \mathrm{O}_{3} 320.1412$, found 320.1410 .

\section{2-(5H-[1,3]Dioxolo[4,5-g]isochromen-8-ylmethyl)-1,2,3,4-tetrahydro-1-naphthal enol (3j):}




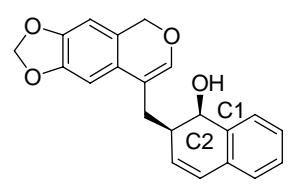

Brownish viscous oil; IR (neat): $3460 \mathrm{~cm}^{-1}(\mathrm{OH}) ;{ }^{1} \mathrm{H}$ NMR (500 MHz, $\left.\mathrm{CDCl}_{3}\right): \delta 7.29$ - 7.18 (m, $4 \mathrm{H}), \quad 7.10$ (d, $J=7.5 \mathrm{~Hz}, 1 \mathrm{H}), 6.88$ (s, $1 \mathrm{H}), 6.56$ - 6.54 (m, $3 \mathrm{H}), 5.91$ 5.89 (m, 2 H), 4.89 (d, $J=3.5$ Hz, 2 H), 4.59 (d, $J=4.0 \mathrm{~Hz}, 1 \mathrm{H})$ (C1 proton), 2.82 (dd, $J=13.0,6.5 \mathrm{~Hz}, 1 \mathrm{H}$ ), $2.70-2.68$ (m, $1 \mathrm{H}$ ) (C2 Proton), 2.52 (dd, $J=14.0,8.5$ $\mathrm{Hz}, 1 \mathrm{H}) ;{ }^{13} \mathrm{C}$ NMR (125 MHz, $\left.\mathrm{CDCl}_{3}\right): \delta$ 147.5, 145.9, 142.8, 136.4, 132.7, 130.1, 128.7, 127.7, 127.6, 127.2, 126.6, 125.6, 122.5, 113.5, 105.5, 101.9, 101.7, 70.0, 68.4, 38.9, 27.6; HRMS (EI): calcd for $\mathrm{C}_{21} \mathrm{H}_{18} \mathrm{O}_{4} 334.1205$, found 334.1207.

\section{2-[(6,7-dimethoxy-1H-4-isochromenyl)methyl]-1,2,3,4-tetrahydro-1-naphthalen ol (3k):}

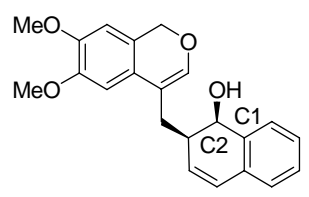

Brownish viscous oil; IR (neat): $3483 \mathrm{~cm}^{-1}(\mathrm{OH}) ;{ }^{1} \mathrm{H}$ NMR (500MHz, $\left.\mathrm{CDCl}_{3}\right): \delta 7.30$ - 7.10 (m, 3 H ), 6.98 (s, 1 H ), 6.73 (s, 1 H), 6.60 - 6.54 (m, 3 H), 5.90 (dd, $J$ = 10.0, $2.4 \mathrm{~Hz}, 1 \mathrm{H}$ ), 4.94 (s, $2 \mathrm{H}$ ), 4.51 (dd, $J=6.4,2.8 \mathrm{~Hz}, 1 \mathrm{H}$ ) (C1 proton), 3.89 (s, $6 \mathrm{H}$ ), 2.85 (dd, $J=15.4,10.0 \mathrm{~Hz}, 1 \mathrm{H}), 2.73-2.70$ (m, $1 \mathrm{H}$ ) (C2 proton), 2.53 (dd, $J=15.4$, $10.0 \mathrm{~Hz}, 1 \mathrm{H}), 1.97$ (t, $\left.J=10.0 \mathrm{~Hz}, 1 \mathrm{H}) ;{ }^{13} \mathrm{C} \mathrm{NMR} \mathrm{(100} \mathrm{MHz,} \mathrm{CDCl}_{3}\right): \delta$ 149.5, 148.9, 142.6, 136.4, 135.2, 132.6, 130.3, 128.6, 127.7, 127.2, 124.2, 121.4, 113.4, 111.6, 108.2, 105.0, 85.3, 69.1, 56.1, 55.9, 39.3, 27.3; HRMS (EI): calcd for $\mathrm{C}_{22} \mathrm{H}_{22} \mathrm{O}_{4}$ 350.1518, found 350.1515.

\section{2-(2-[(4-Methylphenyl)sulfonyl]-1,2-dihydro-4-isoquinolinylmethyl)-1,2,3,4-tetr ahydro-1-naphthalenol (3l):}




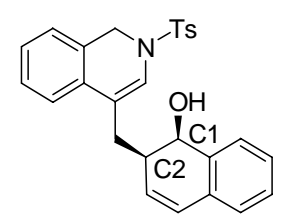

Brownish viscous oil; IR (neat): $3399 \mathrm{~cm}^{-1}(\mathrm{OH}) ;{ }^{1} \mathrm{H}$ NMR (500 MHz, $\left.\mathrm{CDCl}_{3}\right): \delta 7.69$ (d, $J=8.5 \mathrm{~Hz}, 2 \mathrm{H}), 7.26-7.09$ (m, $9 \mathrm{H}$ ), 7.00 (d, $J=7.5 . \mathrm{Hz}, 1 \mathrm{H}), 6.76$ (s, $1 \mathrm{H}$ ), 6.54 (dd, $J=9.5,2.5 . \mathrm{Hz}, 1 \mathrm{H}), 5.79$ (dd, $J=9.5,1.5 . \mathrm{Hz}, 1 \mathrm{H}$ ), 5.27 (s, 2 H), 4.52 (s, $1 \mathrm{H})(\mathrm{C} 1$ proton), 2.95 (dd, $J=13.0,6.5 \mathrm{~Hz}, 1 \mathrm{H}), 2.67$ - $2.58(\mathrm{~m}, 2 \mathrm{H})(\mathrm{C} 2$ proton and a methylene proton), 2.37 (s, $3 \mathrm{H}) ;{ }^{13} \mathrm{C}$ NMR (125 MHz, $\left.\mathrm{CDCl}_{3}\right): \delta$ 143.9, 136.4, 134.5, 132.6, 130.9, 129.9, 129.9, 128.7, 128.6, 127.9, 127.7, 127.6, 127.3, 127.2, 126.6, 125.8, 124.3, 121.7, 119.1, 69.9, 47.7, 38.9, 29.3, 21.4; HRMS (EI): calcd for $\mathrm{C}_{27} \mathrm{H}_{25} \mathrm{NO}_{3} \mathrm{~S}$ 443.1555, found 443.1553.

\section{References:}

1. Perrin, D. D.; Armarego, W. L. F. In Purification of Laboratory Chemicals, 3rd ed.; Pergamon Press: New York, 1988.

2. Grigg, R.; Sansano, J. S. Tetrahedron. 1996, 52, 13441.

3. Stringer, M. B.; Wege, D.; Tetrahedron Lett. 1980, 21, 3831..

4. Colquhoum, H. M.; Halton, J.; Thompson, D. J.; Twigg, M. V. New Pathways for Organic Synthesis-Practical Applications of Transition Metals; Plenum Press: New York, 1988.

5. Heck, R. F. Palladium Reagents in Organic Syntheses, Academic Press: New York, 1978.

6. Takahashi, Y.; Ito, T.; Sakai, S.; Ishii, Y. J. Chem. Soc., Chem. Commun. 1970, 1065. 
${ }^{1} \mathrm{H}$ NMR spectrum of compound 3a:

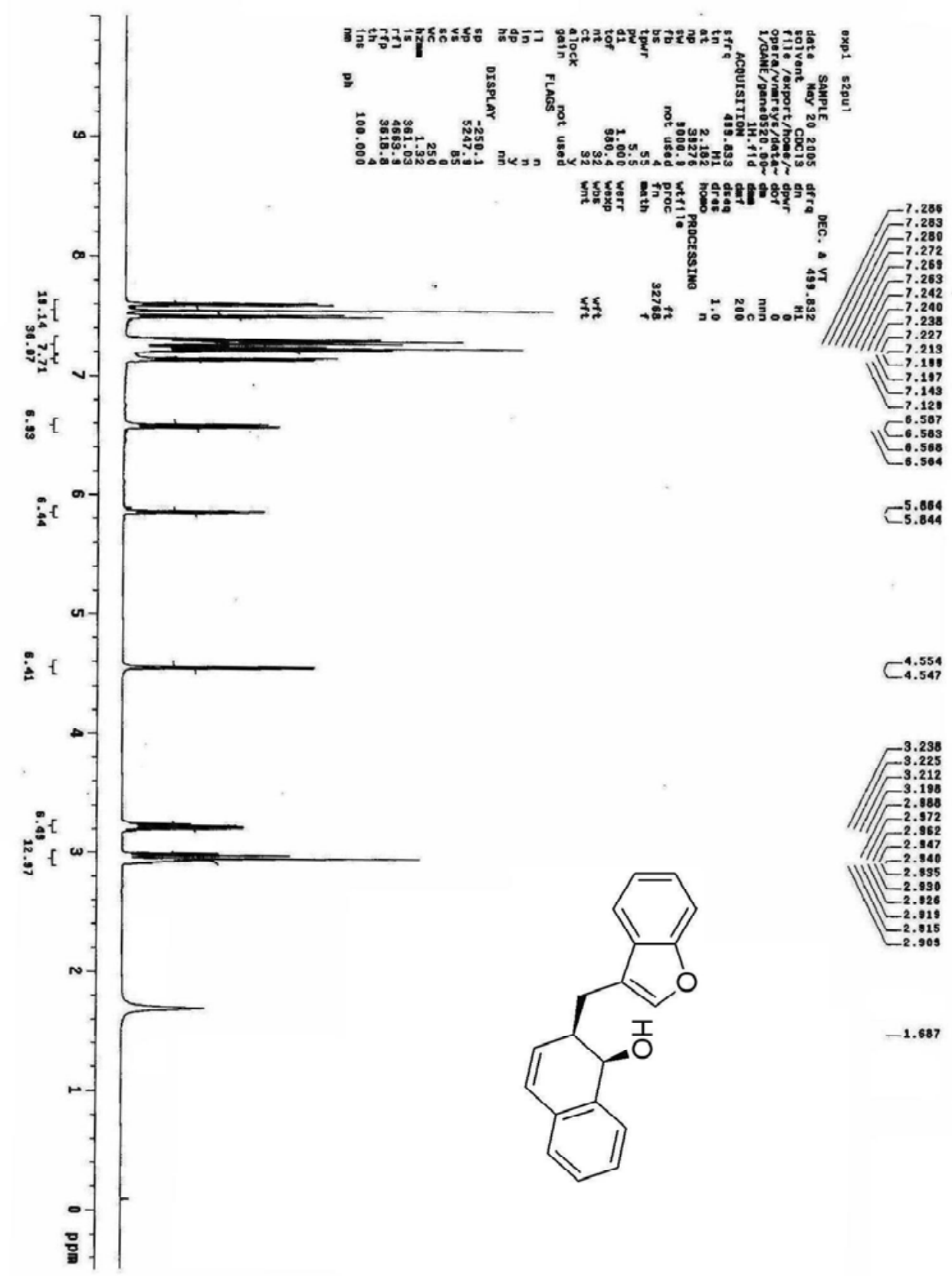


${ }^{13} \mathrm{C}$ NMR spectrum of compound 3a:

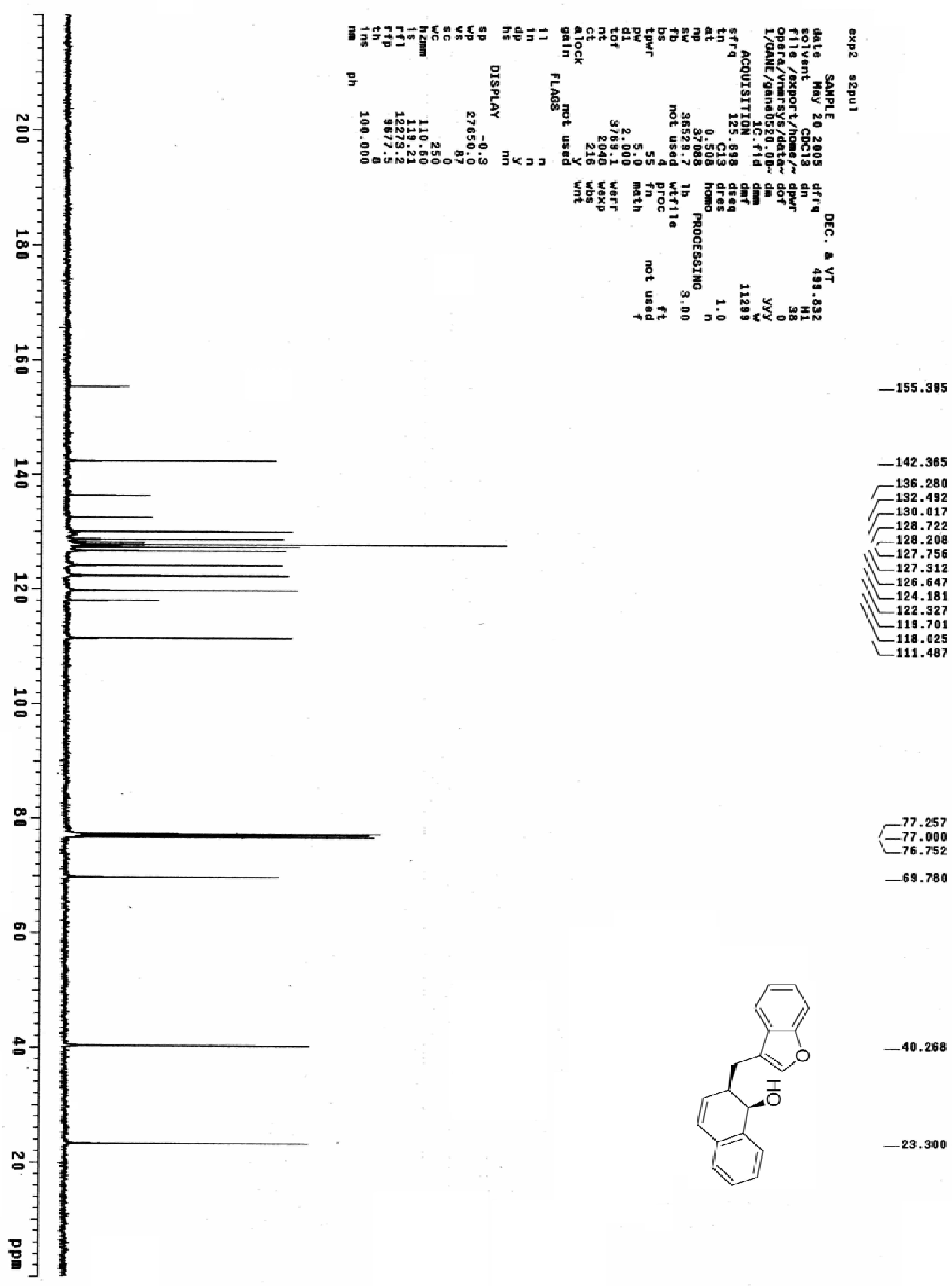


${ }^{1} \mathrm{H}$ NMR spectrum of compound $\mathbf{3 b}$ :

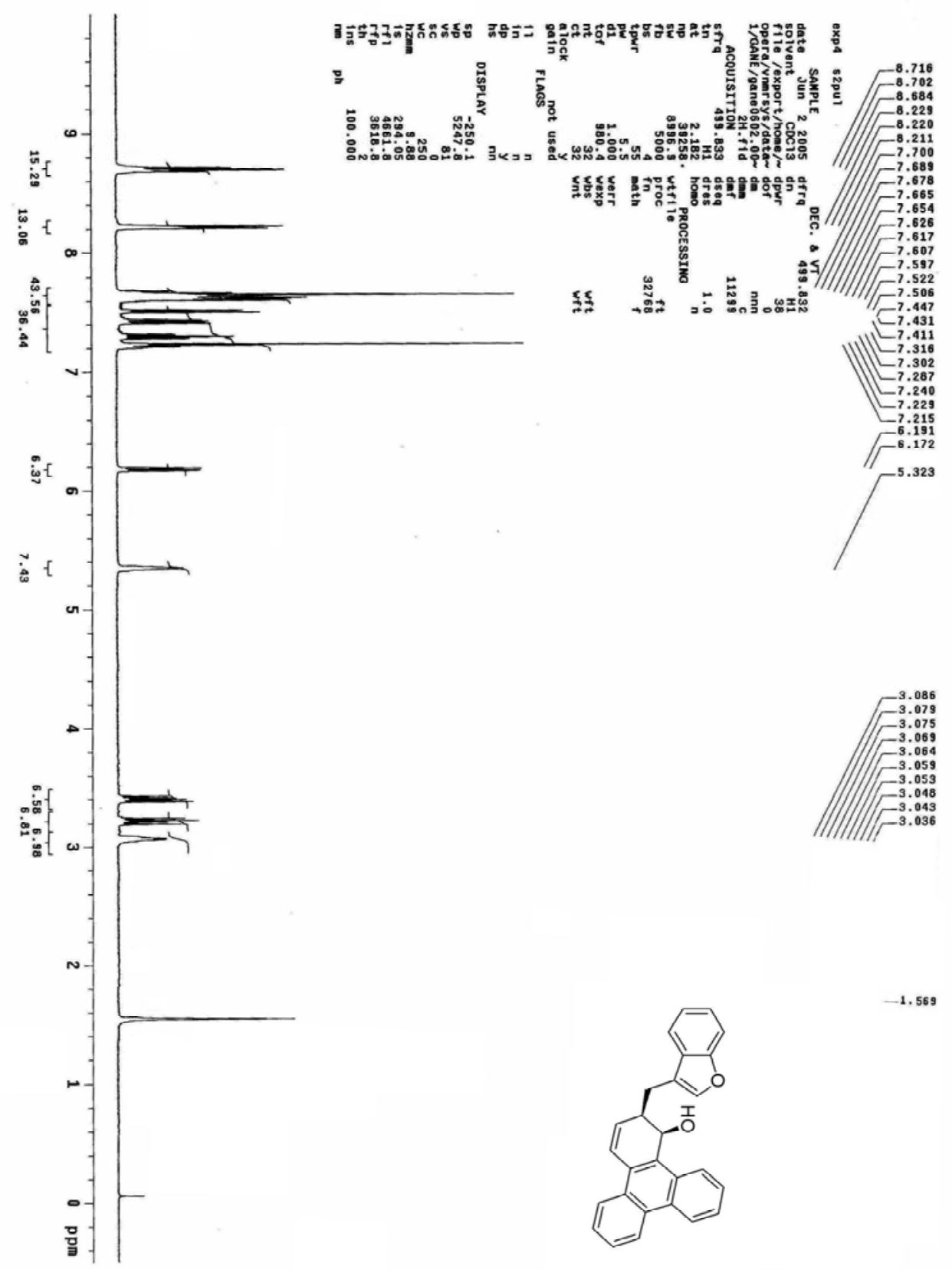


${ }^{13} \mathrm{C}$ NMR spectrum of compound 3b:

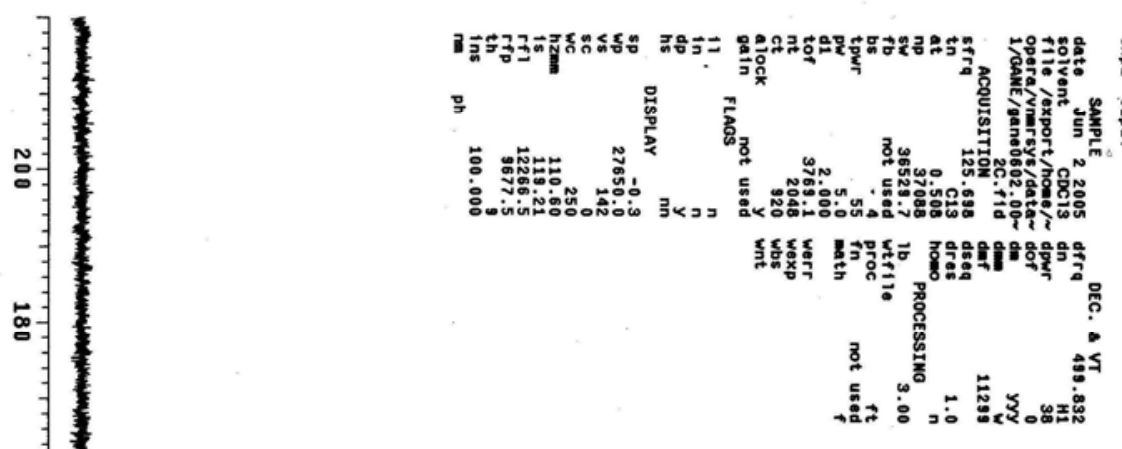

$-142.507$

解

‥-

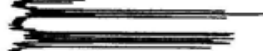

$-131.020$

130.594
-129.751

$-128.643$

$-126.904$

$-124.296$

-124.074
-123.657

政3

$L_{112.9684}^{122.451}$

$\leftarrow_{111.576}^{118.105}$

응

$\infty=$

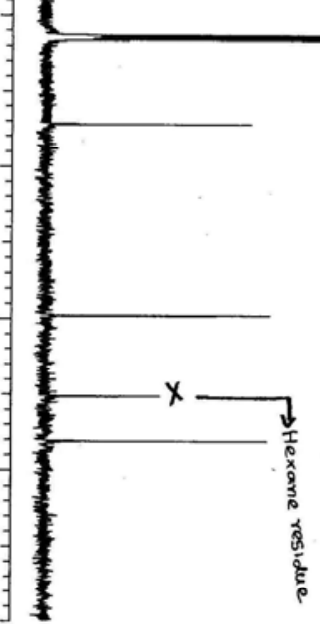

$E_{76.752}^{77.257}$

$-65.531$

$-40.357$

(1)

$-29.695$

$-23.752$ 
Expansion of ${ }^{13} \mathrm{C}$ NMR spectrum of compound $\mathbf{3 b}$ :
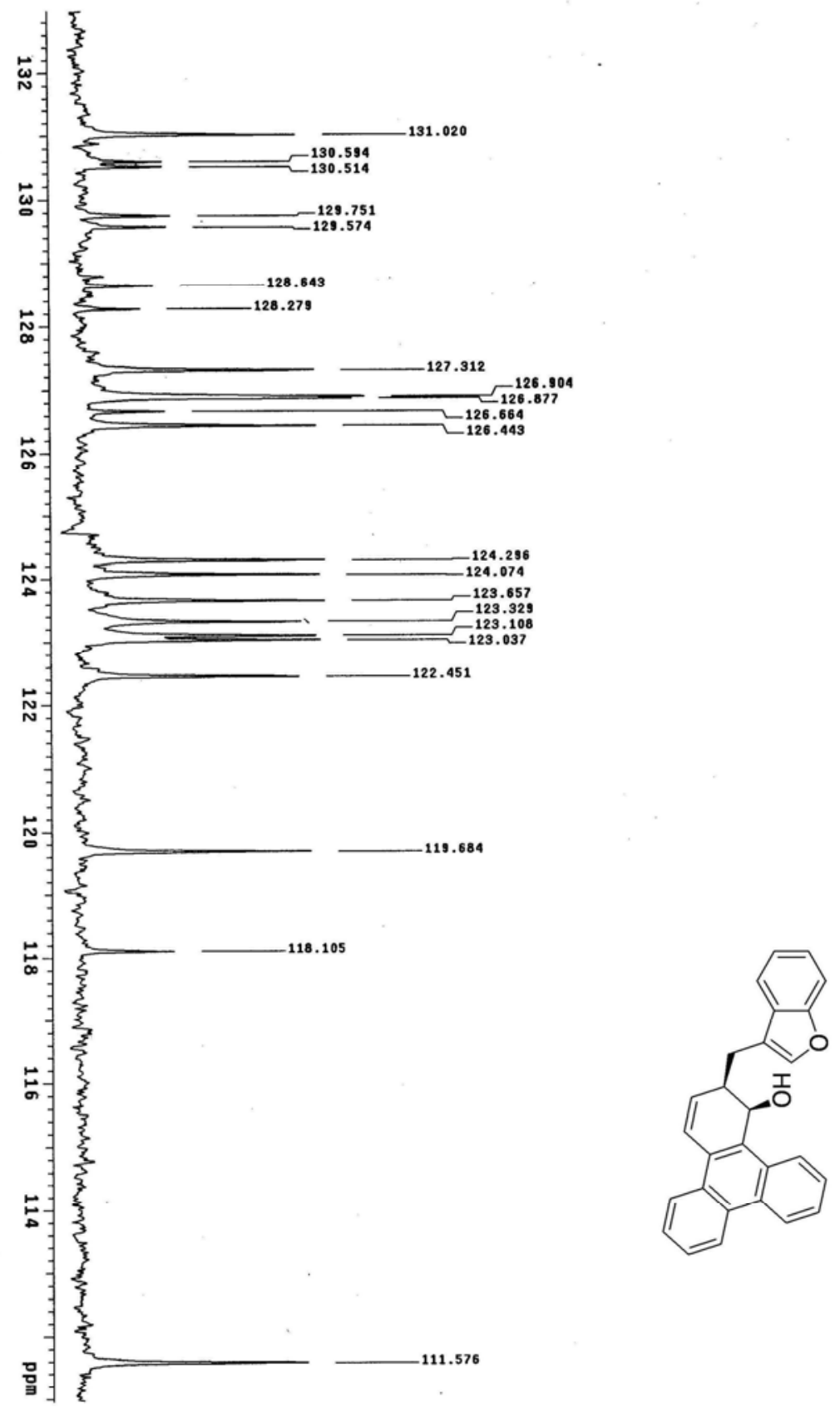
${ }^{1} \mathrm{H}$ NMR spectrum of compound 3c:

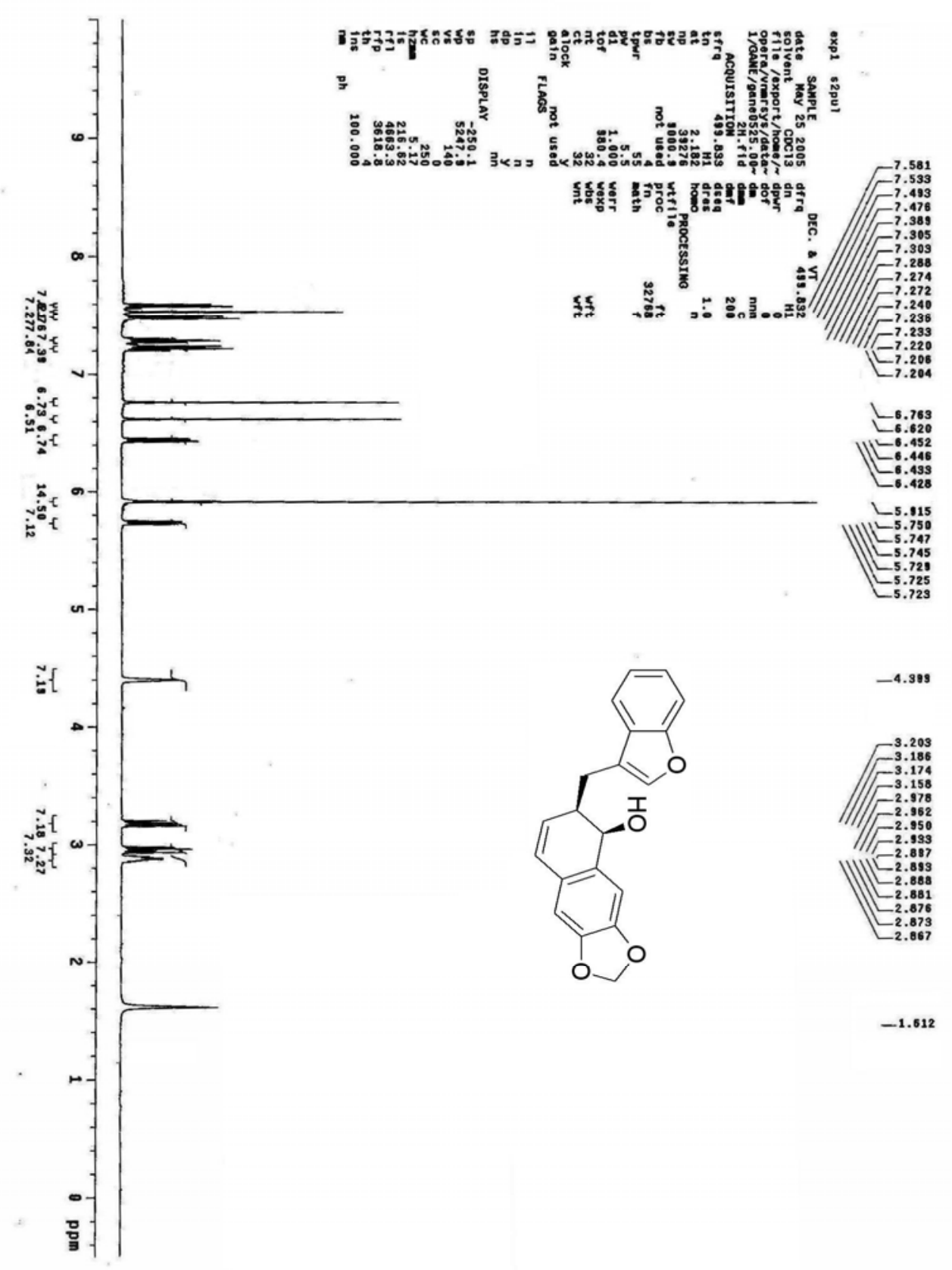


${ }^{13} \mathrm{C}$ NMR spectrum of compound 3c:
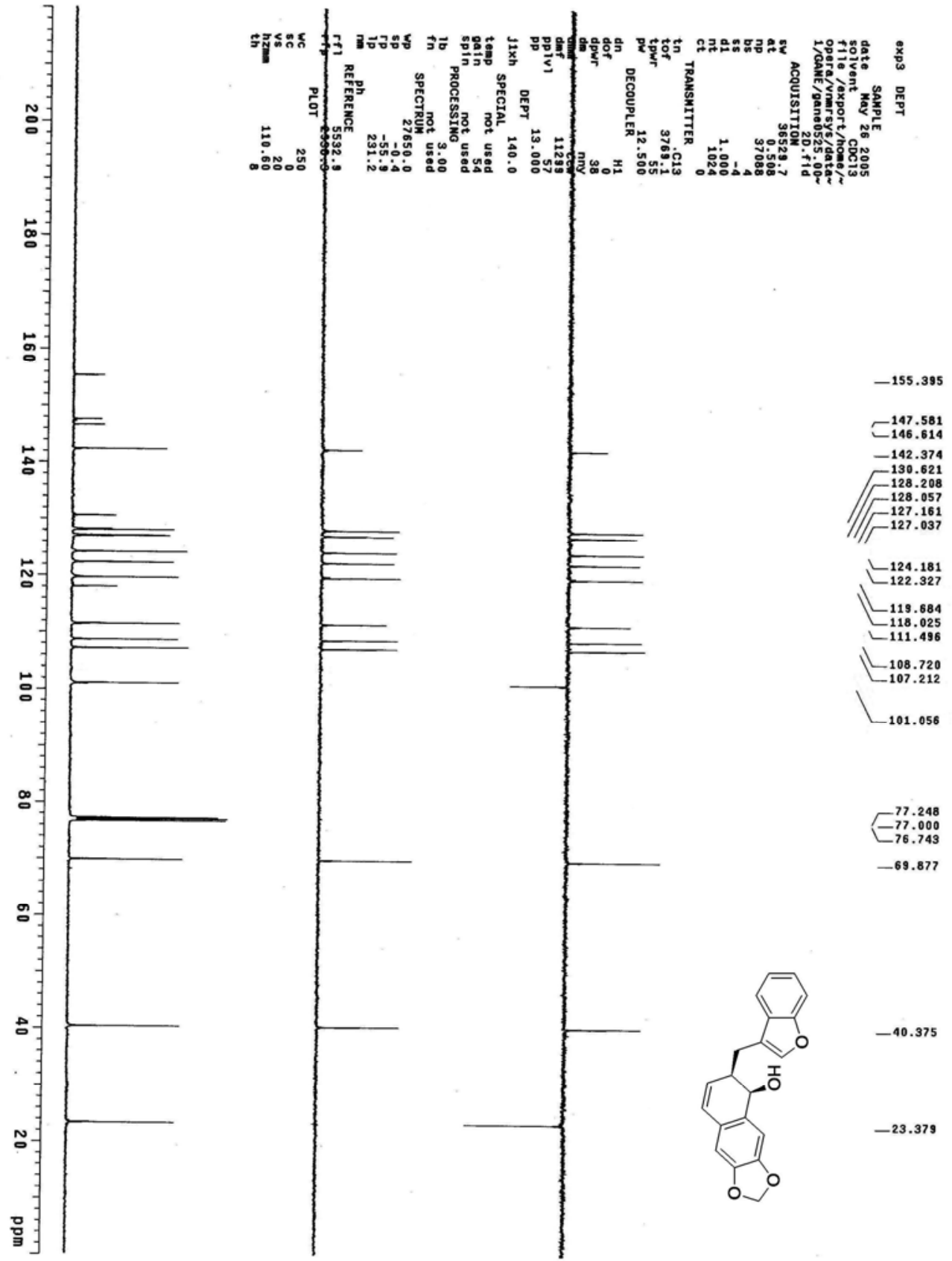
${ }^{1} \mathrm{H}$ NMR spectrum of compound 3d:

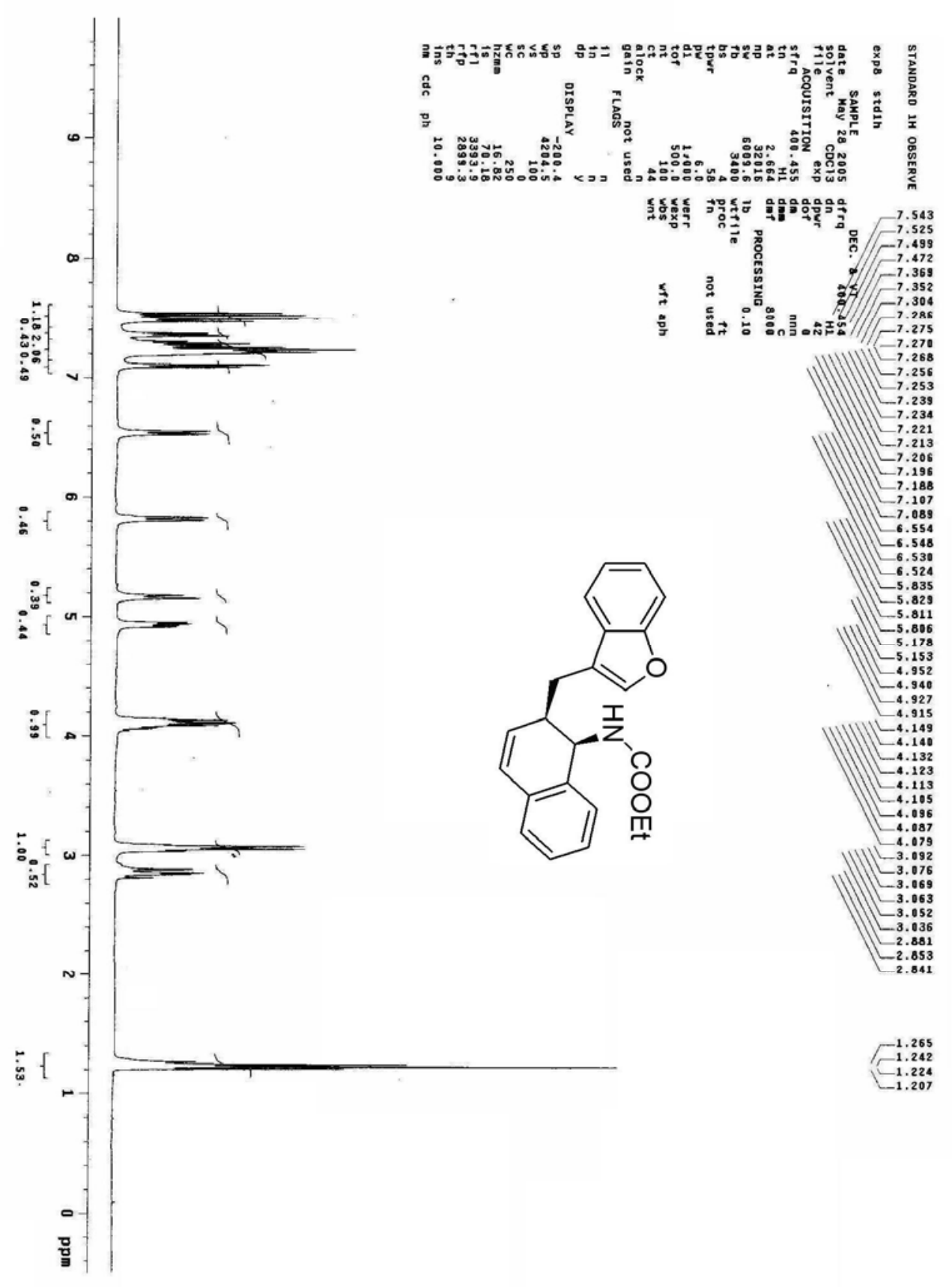


${ }^{13}$ C NMR spectrum of compound 3d:
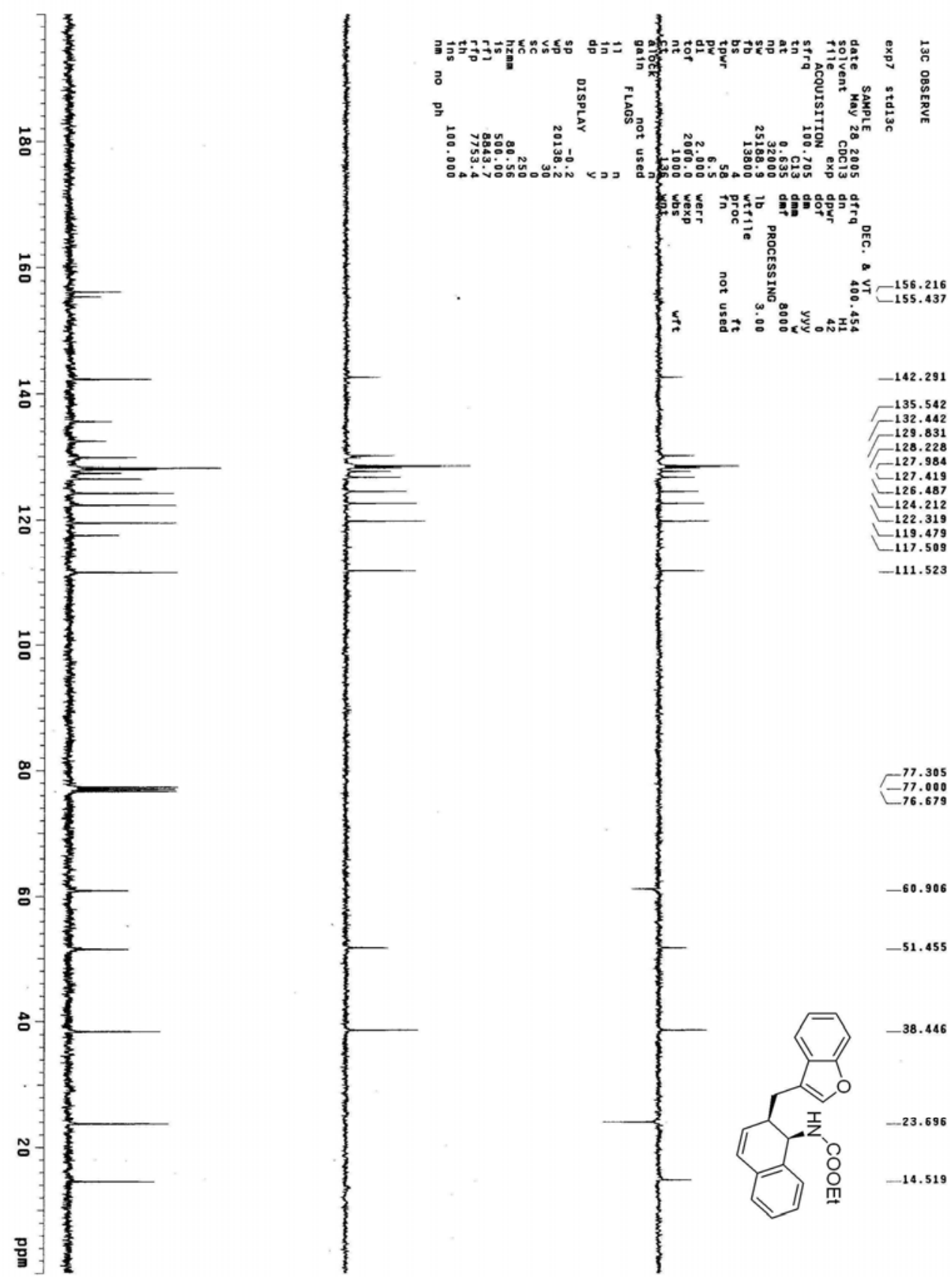
${ }^{1} \mathrm{H}$ NMR spectrum of compound $\mathbf{3 e}$ :

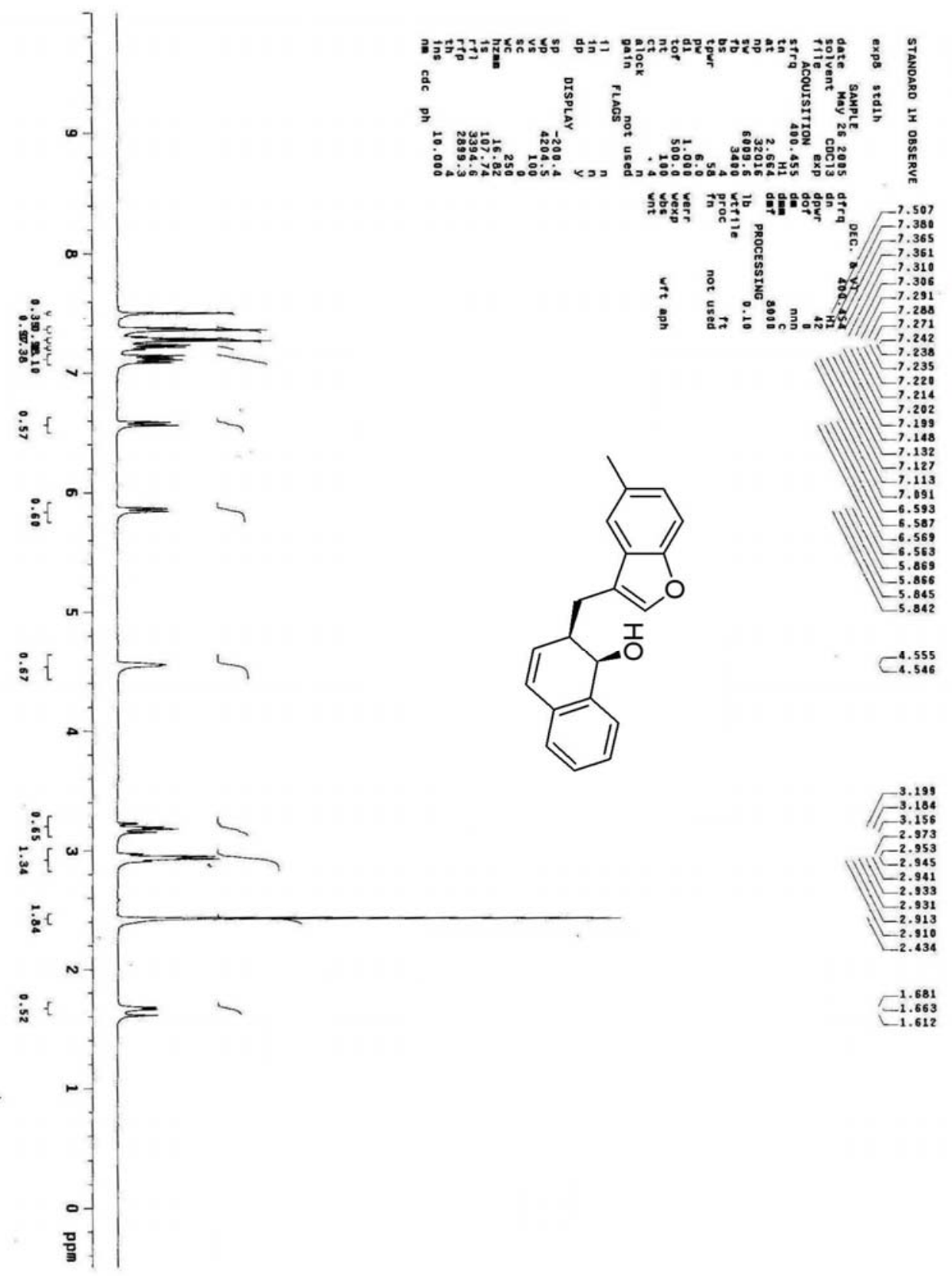


${ }^{13} \mathrm{C}$ NMR spectrum of compound 3e:

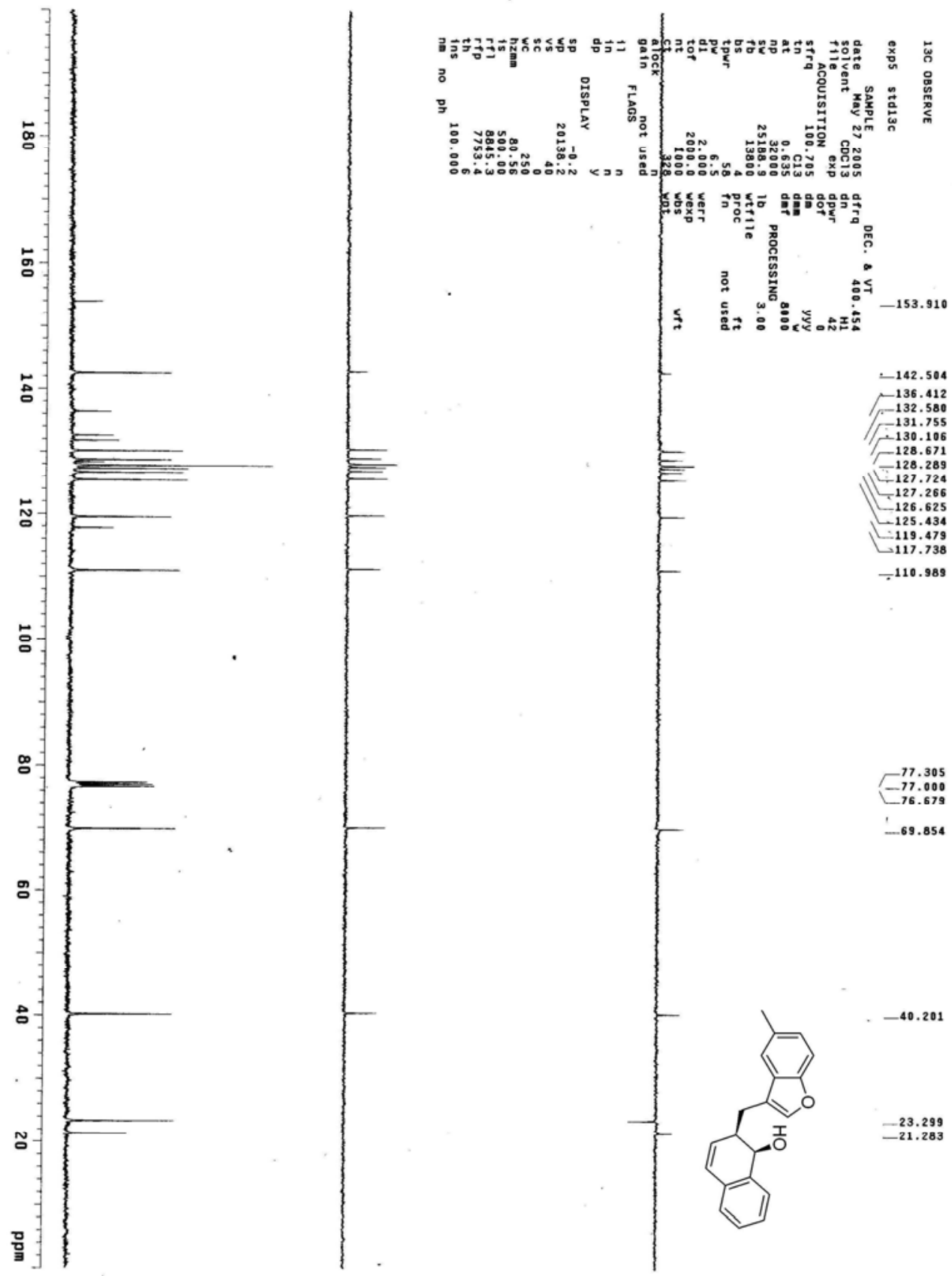


${ }^{1} \mathrm{H}$ NMR spectrum of compound $\mathbf{3 f}$ :

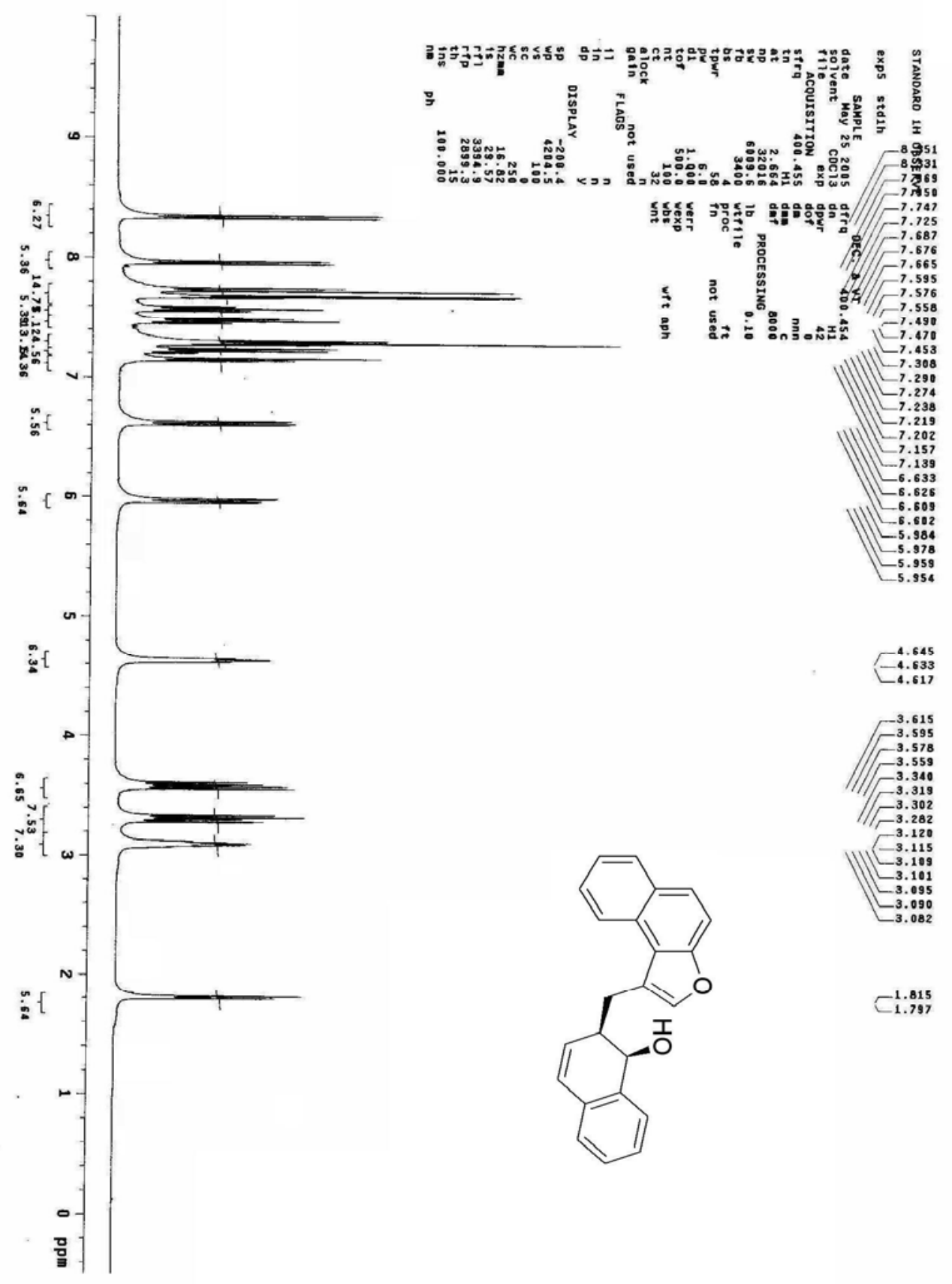


${ }^{13} \mathrm{C}$ NMR spectrum of compound 3f:
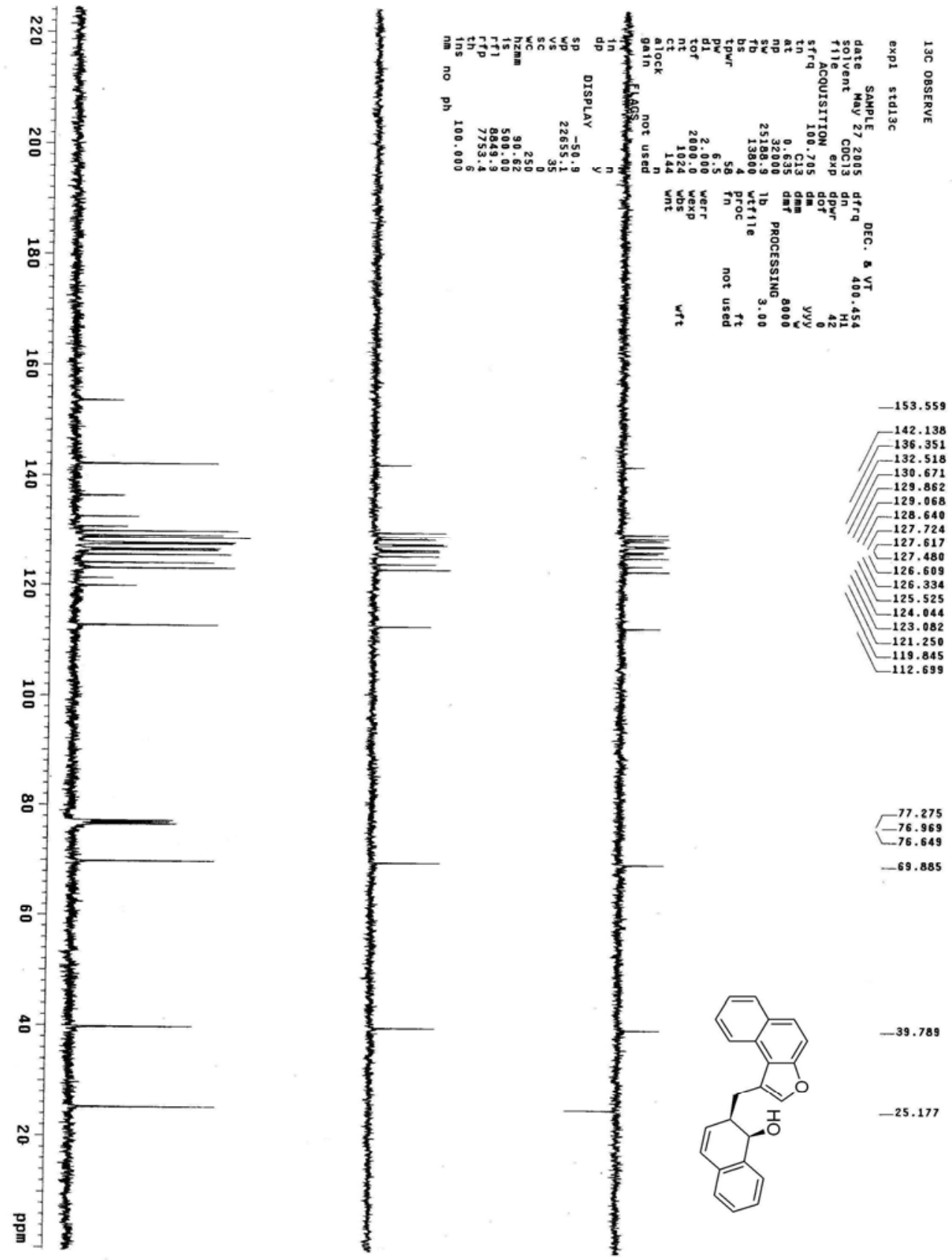
${ }^{1} \mathrm{H}$ NMR spectrum of compound $\mathbf{3 g}$ :

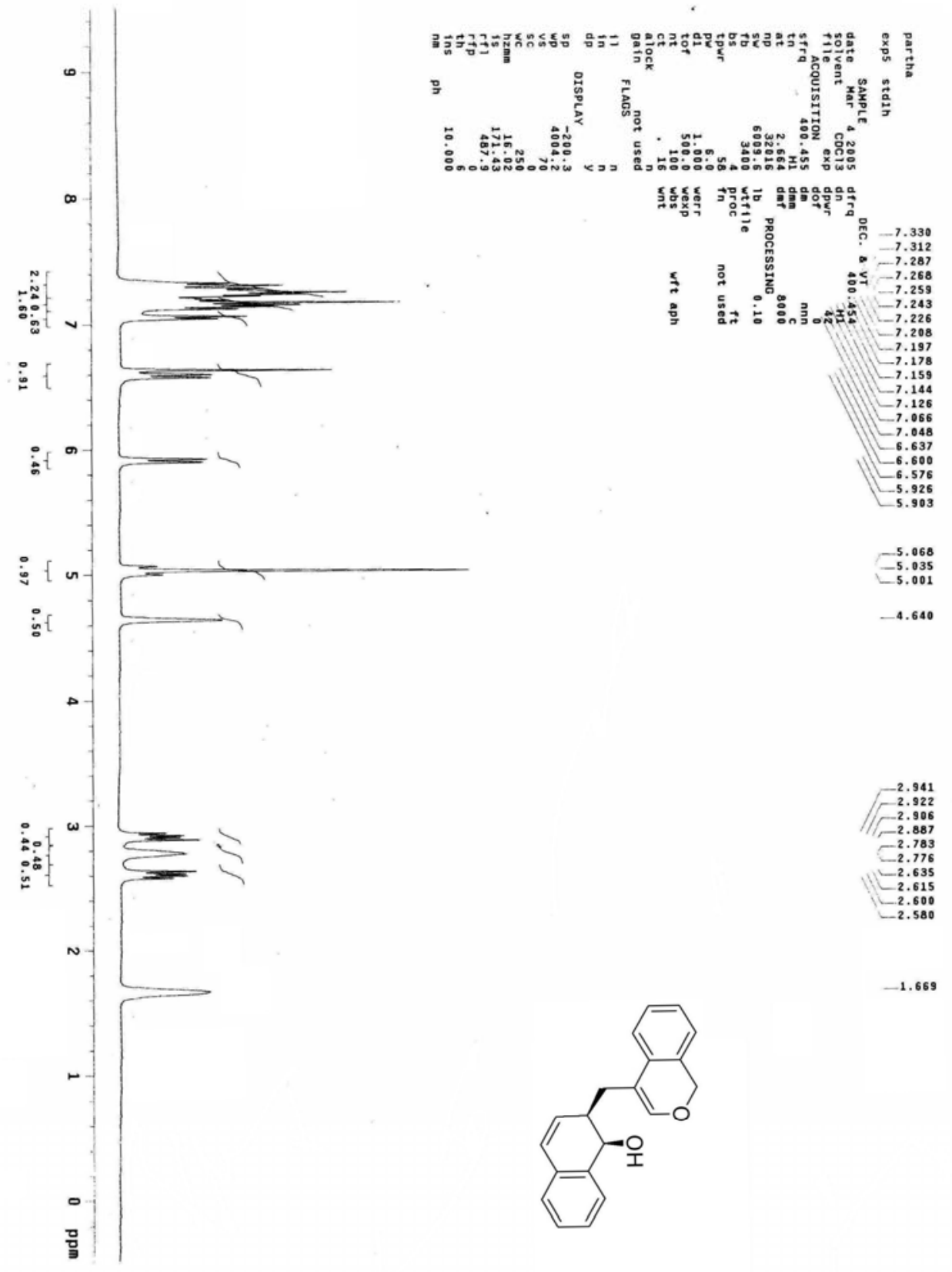


${ }^{13} \mathrm{C}$ NMR spectrum of compound $\mathbf{3 g}$ :

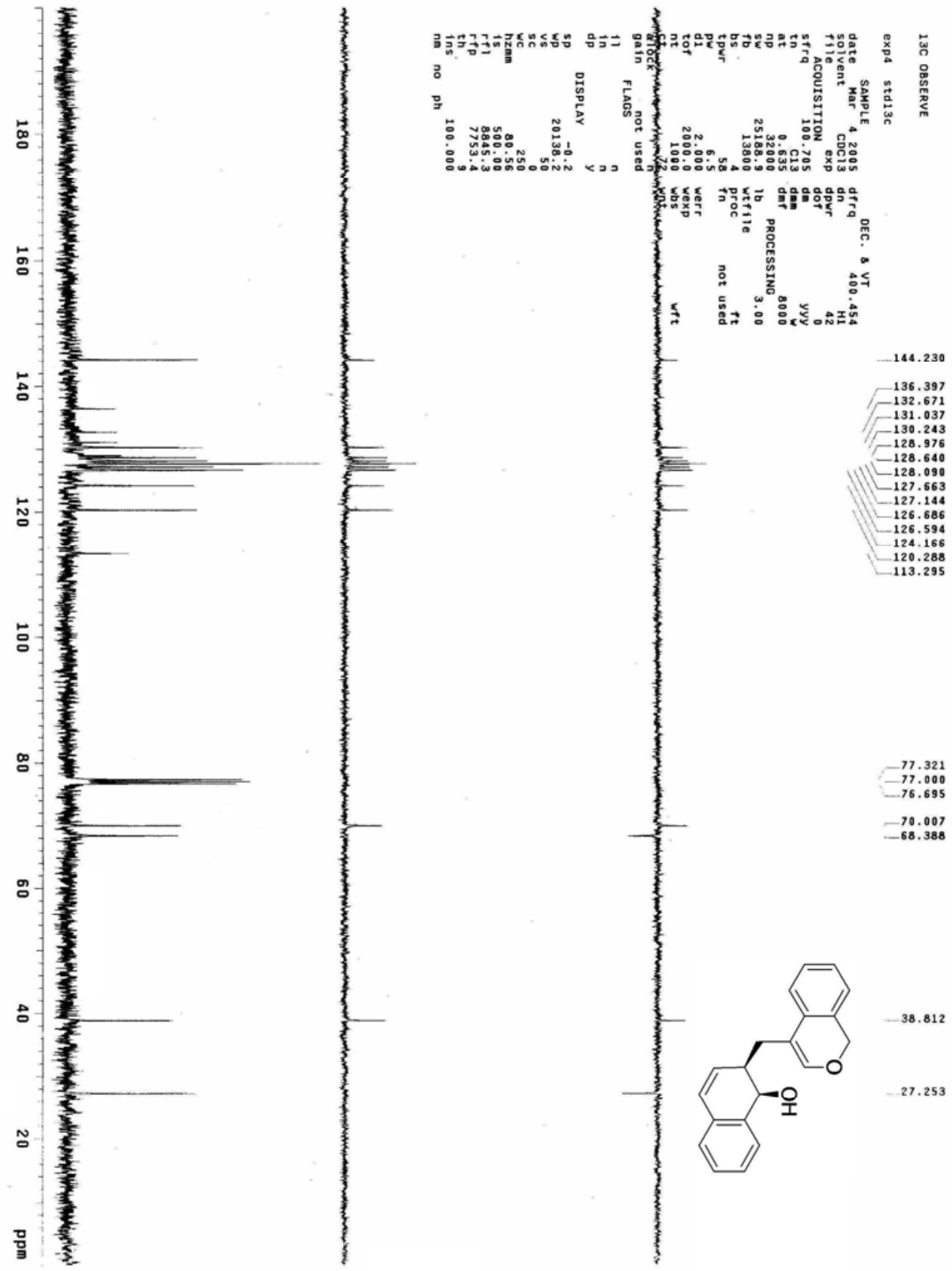


${ }^{1} \mathrm{H}$ NMR spectrum of compound $\mathbf{3 h}$ :

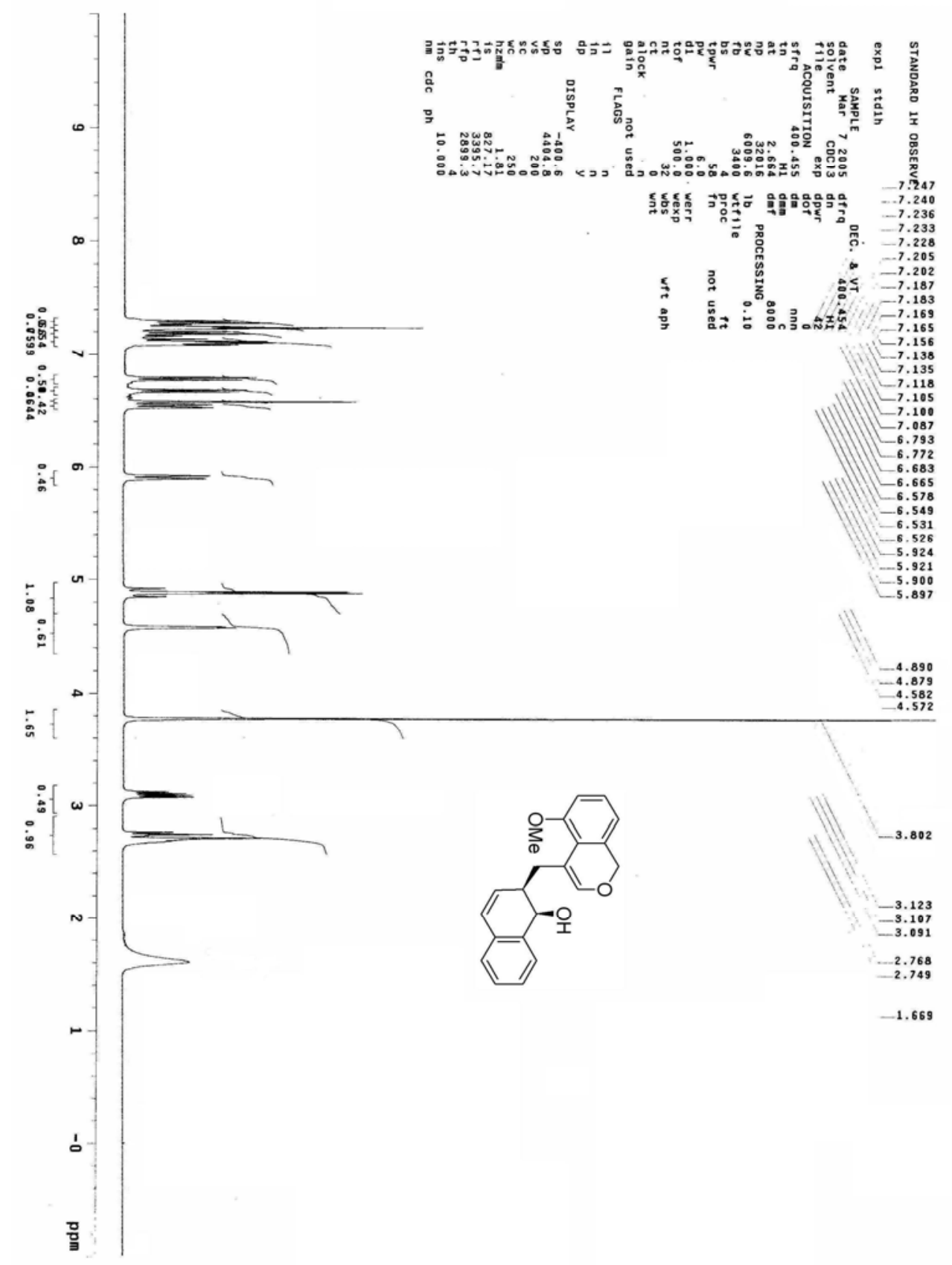


${ }^{13} \mathrm{C}$ NMR spectrum of compound $3 \mathbf{h}$ :
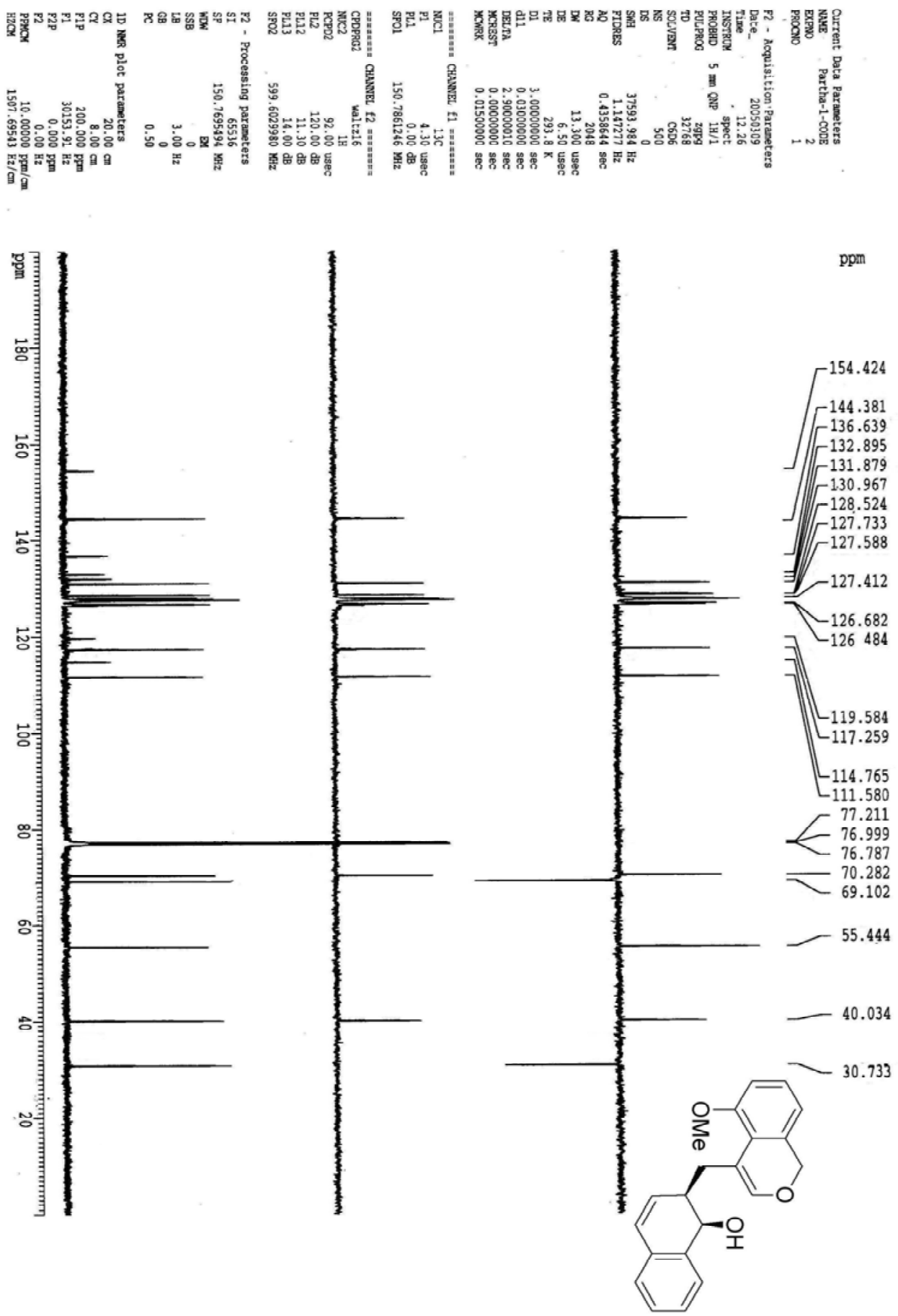
${ }^{1} \mathrm{H}$ NMR spectrum of compound $3 \mathbf{i}$ :

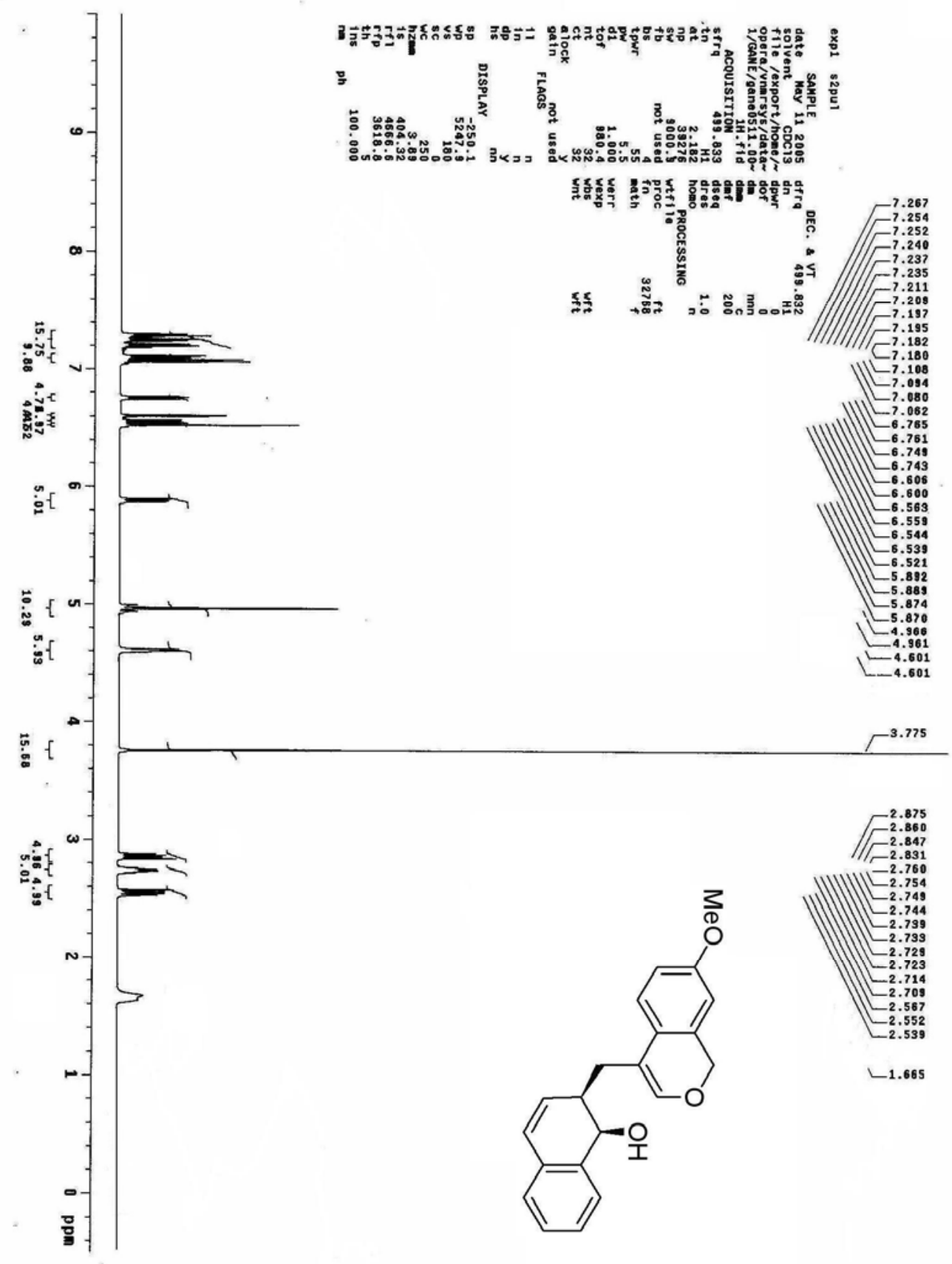


${ }^{13} \mathrm{C}$ NMR spectrum of compound 3i:
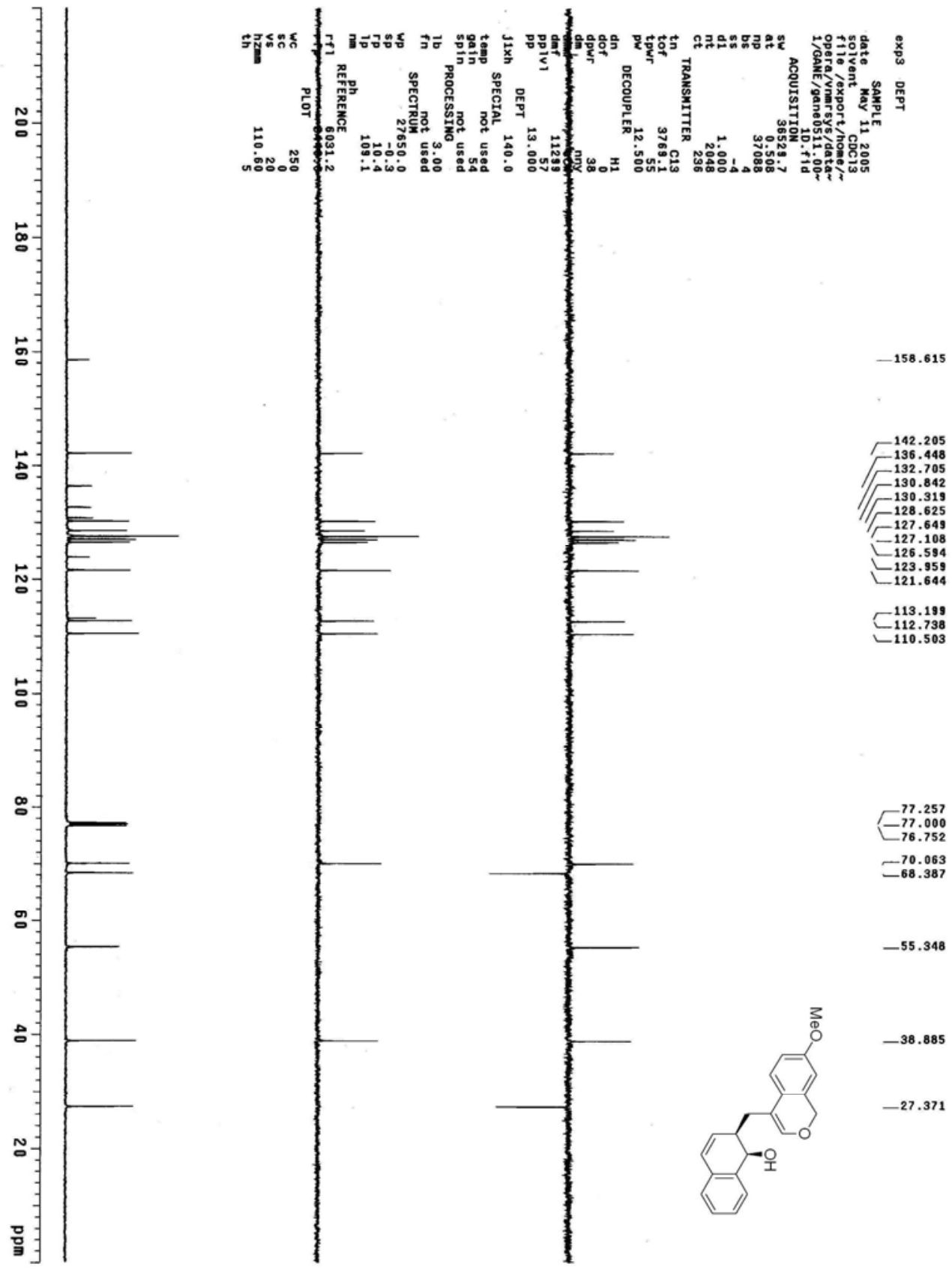
${ }^{1} \mathrm{H}$ NMR spectrum of compound $\mathbf{3 j}$ :

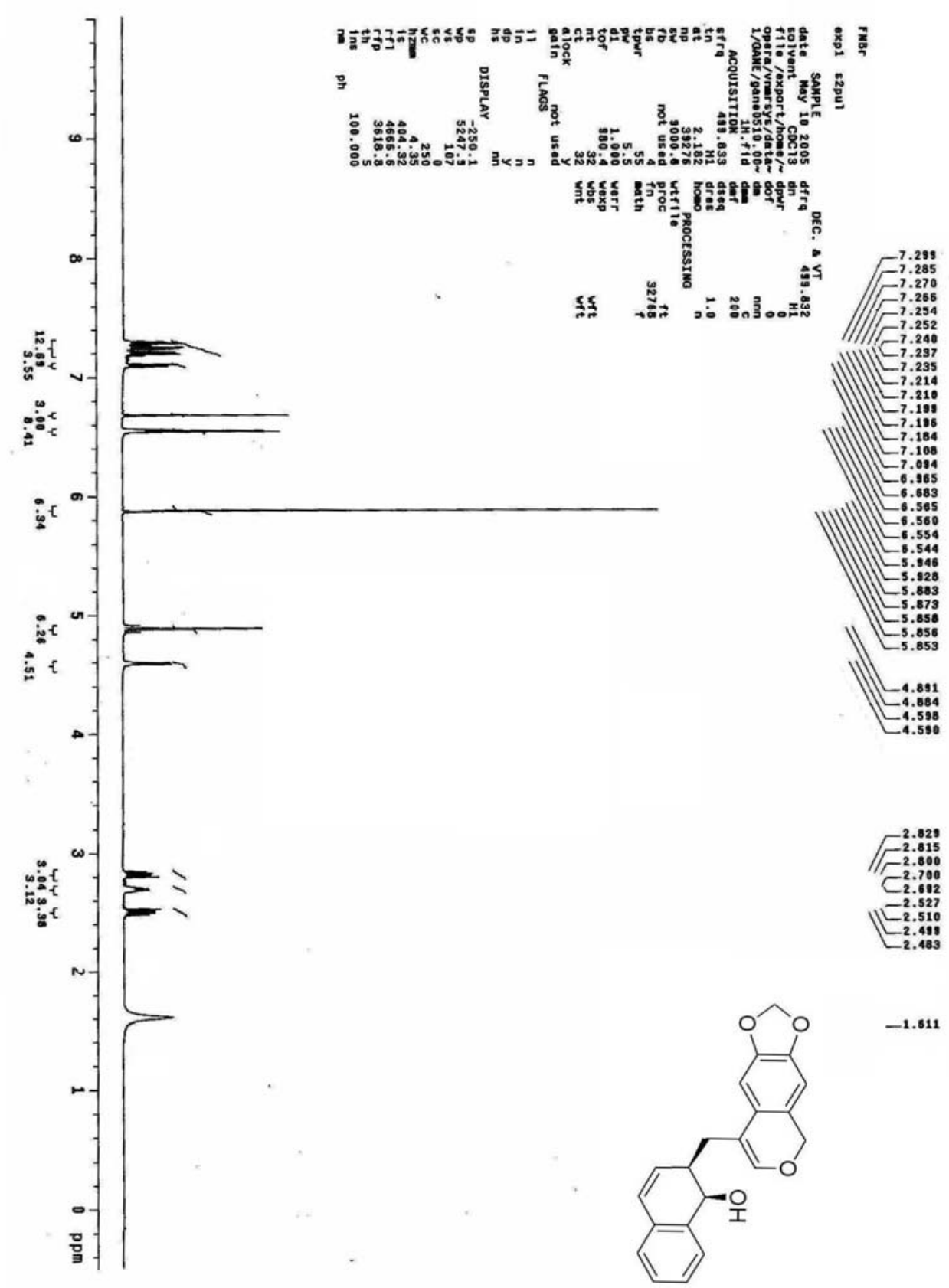


${ }^{13} \mathrm{C}$ NMR spectrum of compound $\mathbf{3 j}$ :

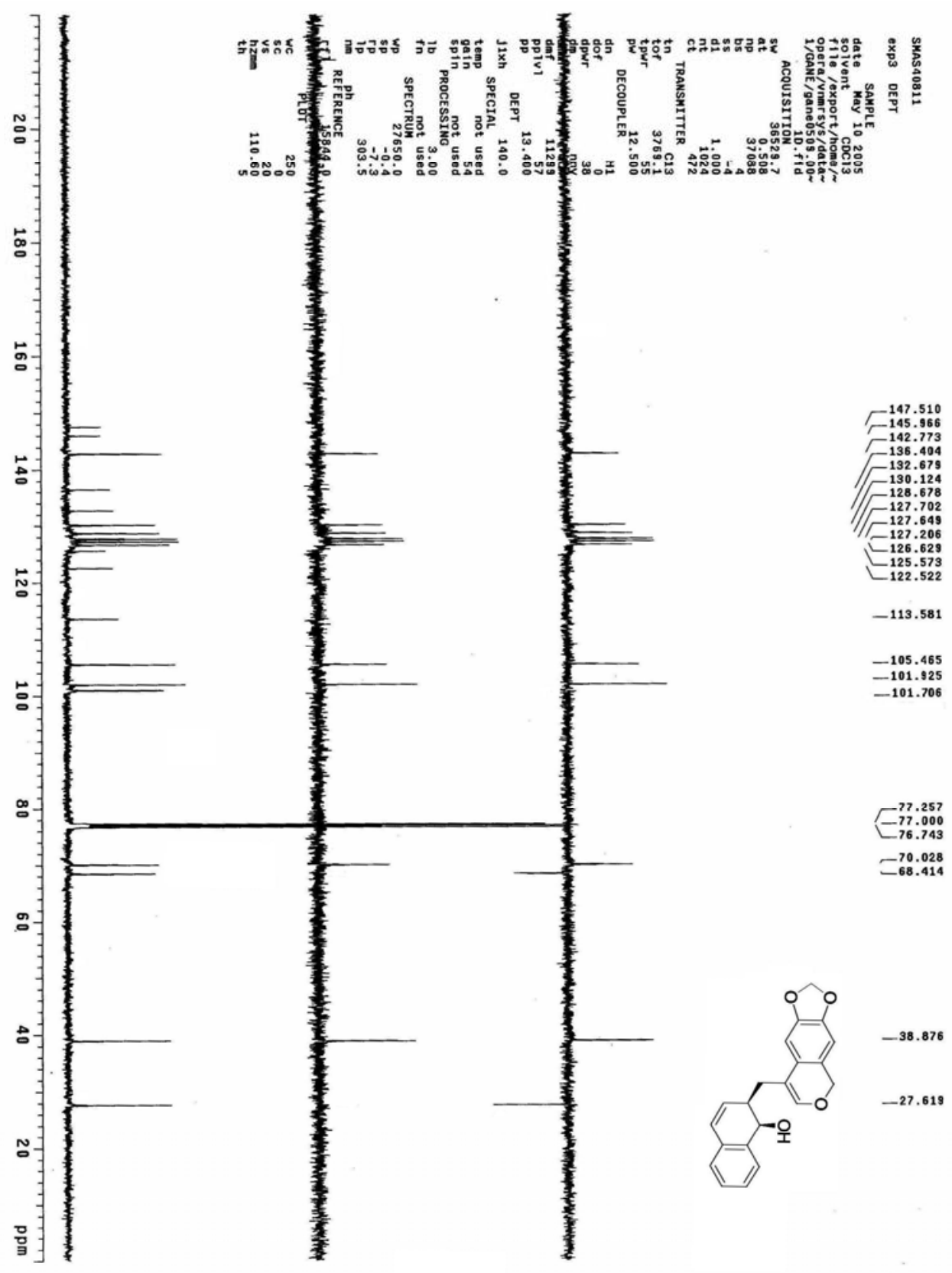


${ }^{1} \mathrm{H}$ NMR spectrum of compound 3k:

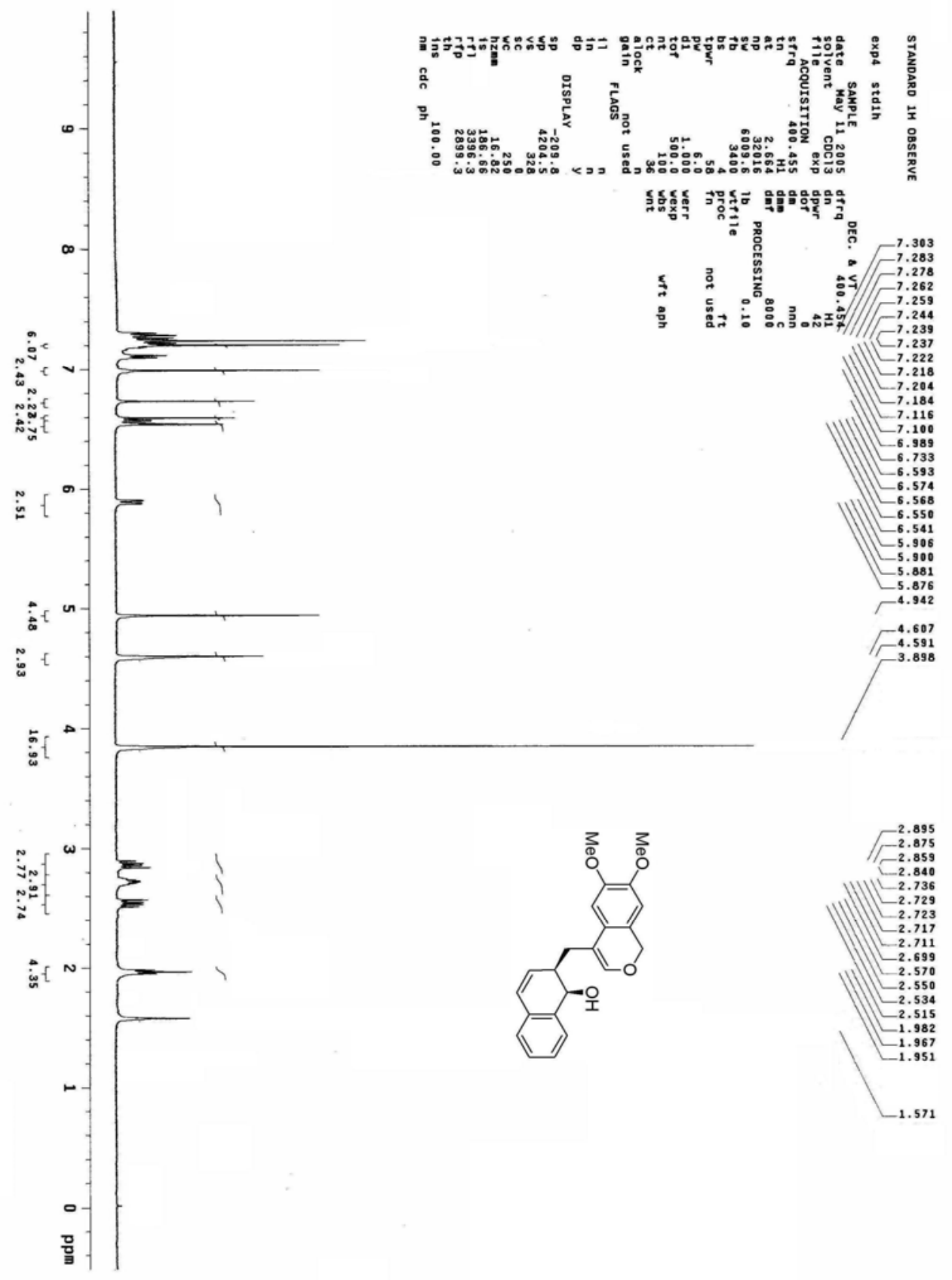


${ }^{13} \mathrm{C}$ NMR spectrum of compound 3k:

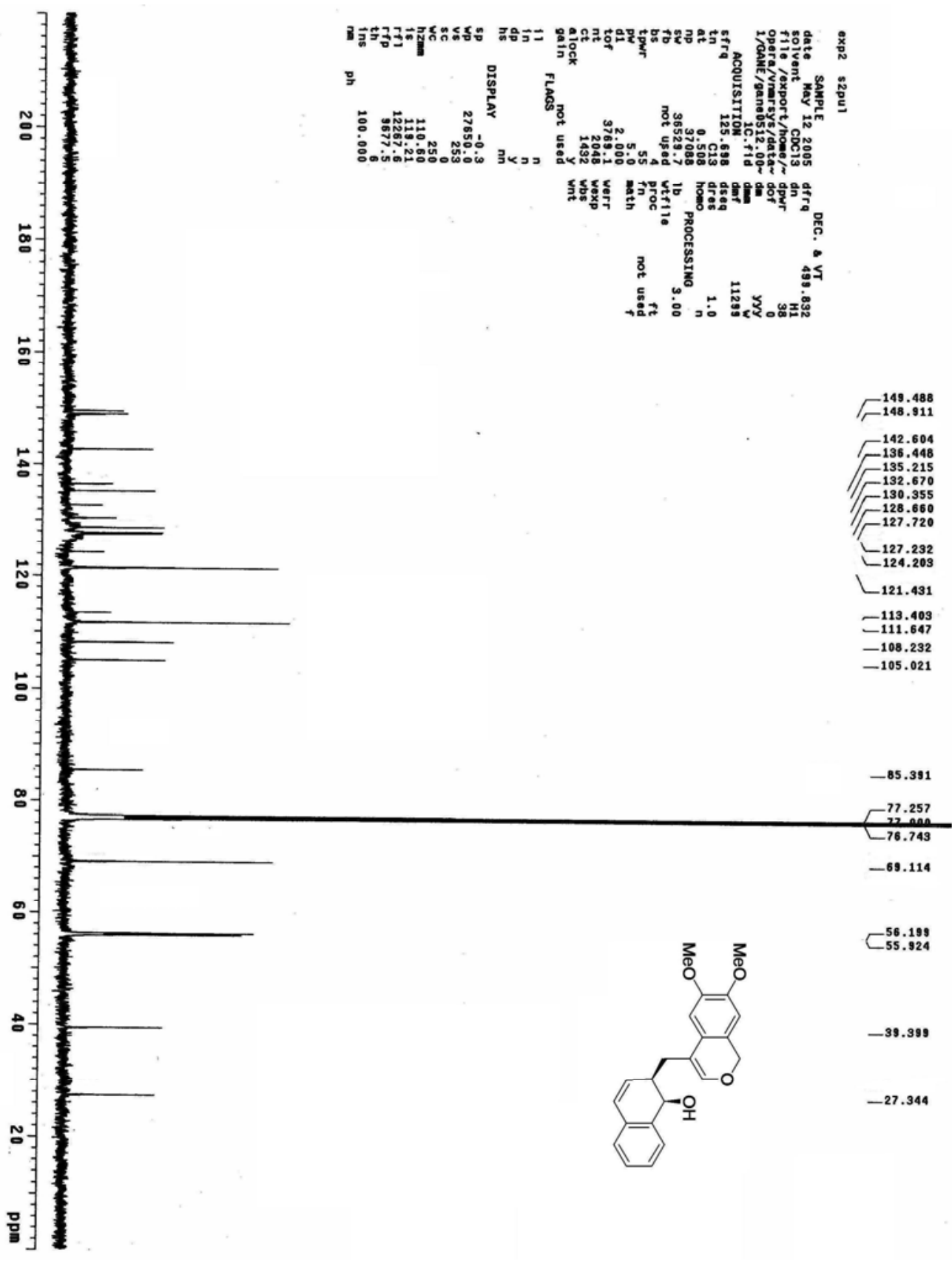


${ }^{1} \mathrm{H}$ NMR spectrum of compound 3l:

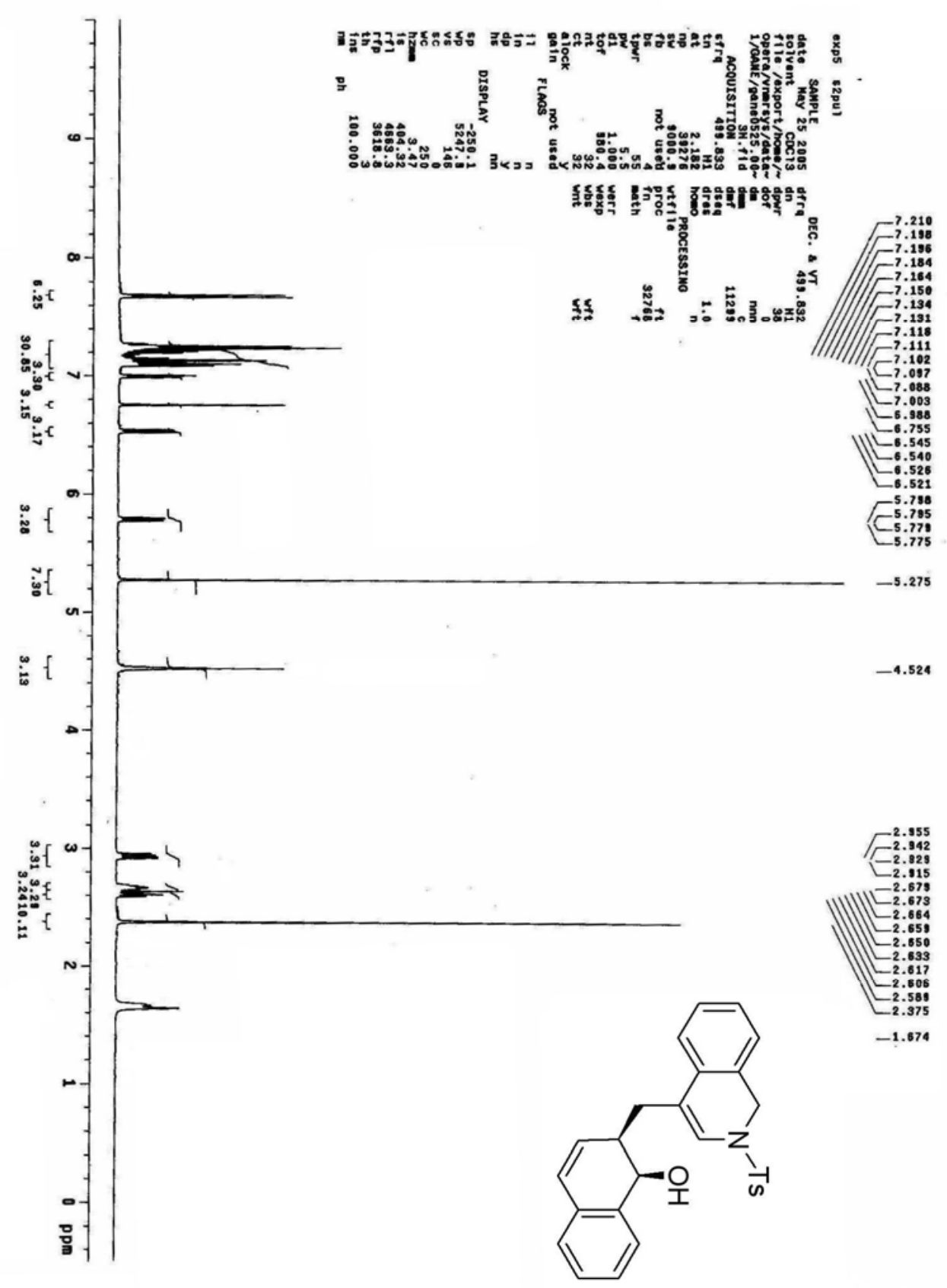


${ }^{13} \mathrm{C}$ NMR spectrum of compound 31:

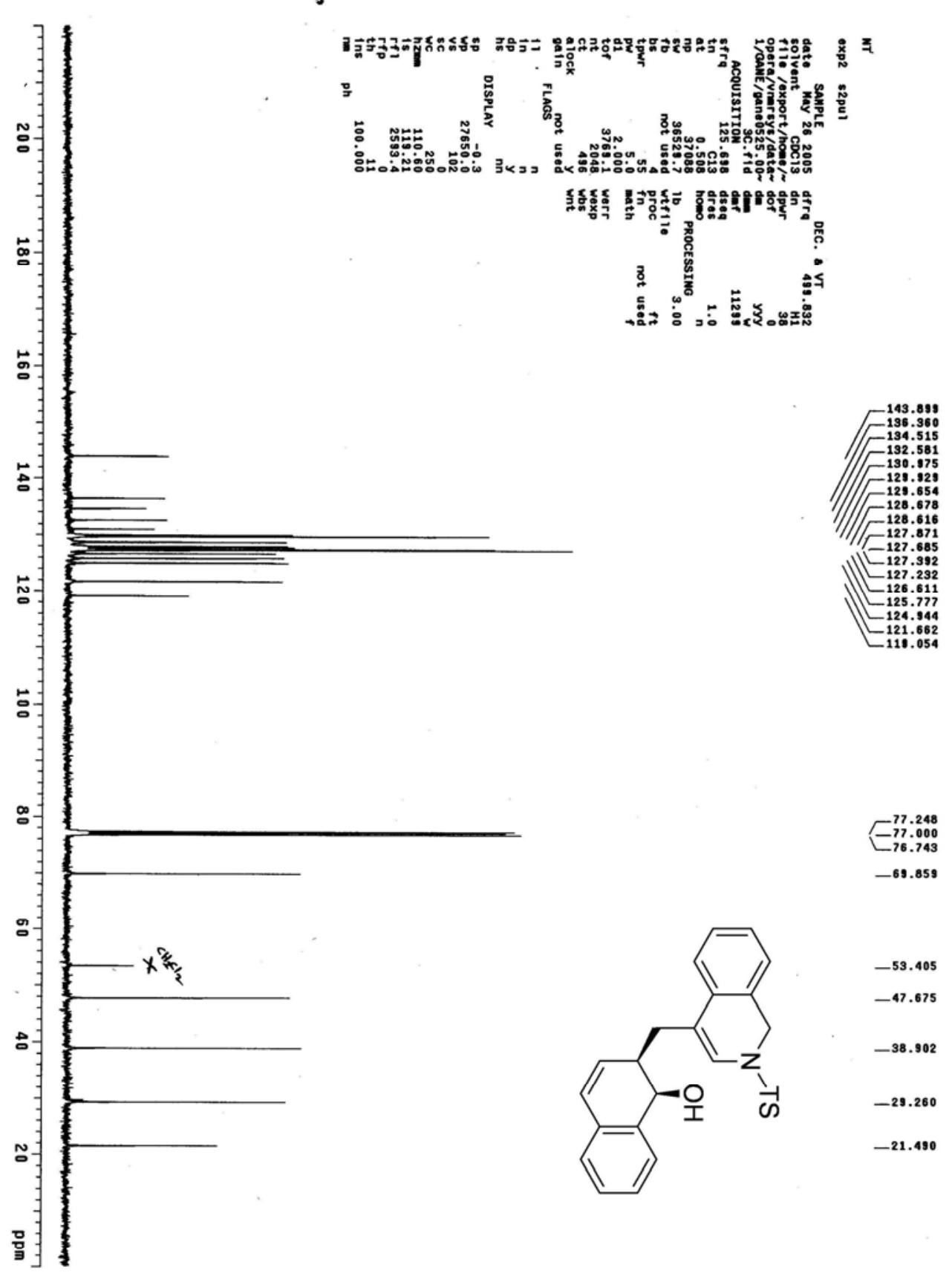


Expansion of ${ }^{13} \mathrm{C}$ NMR spectrum of compound 31:

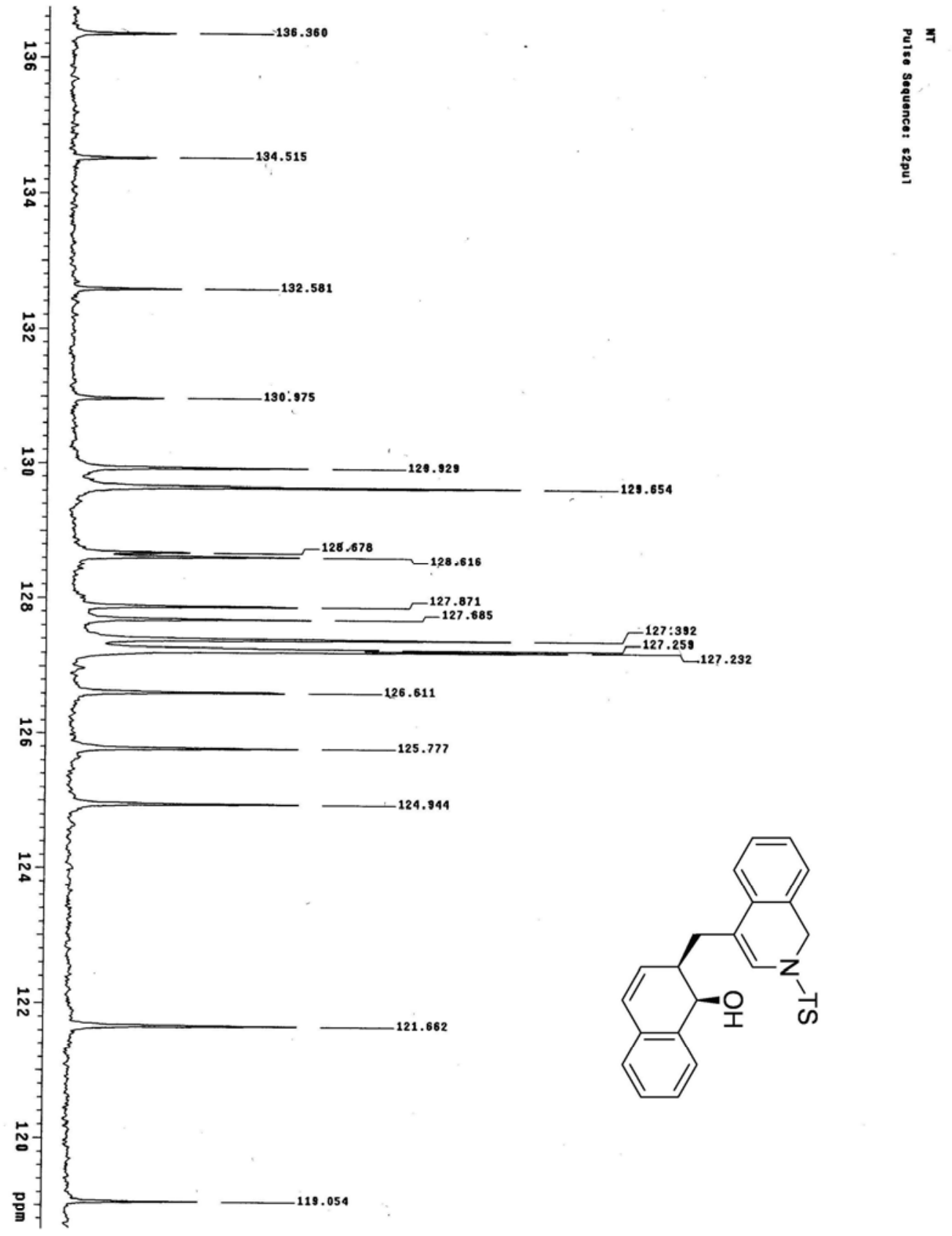

